\title{
ON SOME INEQUALITIES FOR THE GENERALIZED EUCLIDEAN OPERATOR RADIUS
}

\author{
MOHAMMAD W. ALOMARI
}

\begin{abstract}
There are many criterion to generalize the concept of numerical radius; one of the most recent interesting generalization is what so called the generalized Euclidean operator radius. Simply, it is the numerical radius of multivariable operators. In this work, several new inequalities, refinements and generalizations are established for this kind of numerical radius.
\end{abstract}

\section{INTRODUCTION}

Let $\mathscr{B}(\mathscr{H})$ be the Banach algebra of all bounded linear operators defined on a complex Hilbert space $(\mathscr{H} ;\langle\cdot, \cdot\rangle)$ with the identity operator $1_{\mathscr{H}}$ in $\mathscr{B}(\mathscr{H})$. When $\mathscr{H}=\mathbb{C}^{n}$, we identify $\mathscr{B}(\mathscr{H})$ with the algebra $\mathfrak{M}_{n \times n}$ of $n$-by- $n$ complex matrices. Then, $\mathfrak{M}_{n \times n}^{+}$is just the cone of $n$-by- $n$ positive semidefinite matrices.

For a bounded linear operator $T$ on a Hilbert space $\mathscr{H}$, the numerical range $W(T)$ is the image of the unit sphere of $\mathscr{H}$ under the quadratic form $x \rightarrow\langle T x, x\rangle$ associated with the operator. More precisely,

$$
W(T)=\{\langle T x, x\rangle: x \in \mathscr{H},\|x\|=1\}
$$

Also, the numerical radius is defined to be

$$
w(T)=\sup \{|\lambda|: \lambda \in W(T)\}=\sup _{\|x\|=1}|\langle T x, x\rangle| .
$$

We recall that, the usual operator norm of an operator $T$ is defined to be

$$
\|T\|=\sup \{\|T x\|: x \in H,\|x\|=1\} .
$$

It is well known that $w(\cdot)$ defines an operator norm on $\mathscr{B}(\mathscr{H})$ which is equivalent to operator norm $\|\cdot\|$. Moreover, we have

$$
\frac{1}{2}\|T\| \leq w(T) \leq\|T\|
$$

for any $T \in \mathscr{B}(\mathscr{H})$ and this inequality is sharp.

It is known that $w(A)$ is a norm on $\mathscr{B}(\mathscr{H})$, but it is not unitarily invariant. But the numerical radius norm is weakly unitarily invariant; i.e., $w\left(U^{*} T U\right)=w(T)$ for all unitary $U$. Also, let us don't miss the chance to mention the important property that $w(T)=w\left(T^{*}\right)$ and $w\left(T^{*} T\right)=w\left(T T^{*}\right)$ for every $T \in \mathscr{B}(\mathscr{H})$.

Denote $|T|=\left(T^{*} T\right)^{1 / 2}$ the absolute value of the operator $T$. Then we have

$$
w(|T|)=\|T\| .
$$

It's well known that the numerical radius is not submultiplicative, but it satisfies

$$
w(T S) \leq 4 w(T) w(S)
$$

for all $T, S \in \mathscr{B}(\mathscr{H})$. In particular if $T, S$ commute, then

$$
w(T S) \leq 2 w(T) w(S) .
$$

Moreover, if $T, S$ are normal then $w(\cdot)$ is submultiplicative, i.e.,

$$
w(T S) \leq w(T) w(S)
$$

Date: January 1, 2020.

2000 Mathematics Subject Classification. 47A12, 47B15, 47A30, 47A63.

Key words and phrases. Euclidean operator radius, numerical radius, self-adjoint operator. 
In 2009, Popsecu [21] introduced the concept of Euclidean operator radius of an $n$-tuple $\mathbf{T}=\left(T_{1}, \cdots, T_{n}\right) \in$ $\mathscr{B}(\mathscr{H})^{n}:=\mathscr{B}(\mathscr{H}) \times \cdots \times \mathscr{B}(\mathscr{H})$. Namely, for $T_{1}, \cdots, T_{n} \in \mathscr{B}(\mathscr{H})$. The Euclidean operator radius of $T_{1}, \cdots, T_{n}$ is defined by

$$
w_{\mathrm{e}}\left(T_{1}, \cdots, T_{n}\right):=\sup _{\|x\|=1}\left(\sum_{i=1}^{n}\left|\left\langle T_{i} x, x\right\rangle\right|^{2}\right)^{1 / 2} .
$$

Indeed, the Euclidean operator radius was generalized in [24] as follows:

$$
w_{p}\left(T_{1}, \cdots, T_{n}\right):=\sup _{\|x\|=1}\left(\sum_{i=1}^{n}\left|\left\langle T_{i} x, x\right\rangle\right|^{p}\right)^{1 / p}, \quad p \geq 1 .
$$

If $p=1$ then $w_{1}\left(T_{1}, \cdots, T_{n}\right)$ (also, it is denoted by $w_{R}\left(T_{1}, \cdots, T_{n}\right)$ ) is called the Rhombic numerical radius which have been studied in [5]. In an interesting case, $w_{1}(C, \cdots, C)=n \cdot w(C)$.

The Crawford number is defined to be

$$
c(T)=\inf \{|\lambda|: \lambda \in W(T)\}=\inf _{\|x\|=1}|\langle T x, x\rangle| .
$$

Consequently, we define the generalized Crawford number as:

$$
c_{p}\left(T_{1}, \cdots, T_{n}\right):=\inf _{\|x\|=1}\left(\sum_{i=1}^{n}\left|\left\langle T_{i} x, x\right\rangle\right|^{p}\right)^{1 / p}, \quad p \geq 1 .
$$

In case $p=1$, the generalized Crawford number is called the Rhombic Crawford number and is denoted by $c_{R}\left(T_{1}, \cdots, T_{n}\right)$.

We note that in case $p=\infty$, the generalized Euclidean operator radius is defined as:

$$
\begin{aligned}
w_{\infty}\left(T_{1}, \cdots, T_{n}\right) & :=\sup _{\|x\|=1} \sum_{i=1}^{n}\left|\left\langle T_{i} x, x\right\rangle\right|-\inf _{\|x\|=1} \sum_{i=1}^{n}\left|\left\langle T_{i} x, x\right\rangle\right| \\
& =w_{R}\left(T_{1}, \cdots, T_{n}\right)-c_{R}\left(T_{1}, \cdots, T_{n}\right) .
\end{aligned}
$$

Thus, the inequality

$$
w_{\infty}\left(T_{1}, \cdots, T_{n}\right) \leq w_{p}\left(T_{1}, \cdots, T_{n}\right) \leq w_{R}\left(T_{1}, \cdots, T_{n}\right)
$$

for all $p \in(1, \infty)$. This fact follows by Jensen's inequality applied for the function $h(p)=w_{p}\left(T_{1}, \cdots, T_{n}\right)$, which is log-convex and decreasing for all $p>1$.

On the other hand, by employing the Jensen's inequality

$$
\left(\frac{1}{n} \sum_{k=1}^{n} a_{k}\right)^{p} \leq \frac{1}{n} \sum_{k=1}^{n} a_{k}^{p}
$$

which holds for every finite positive sequence of real numbers $\left(a_{k}\right)_{k=1}^{n}$ and $p \geq 1$; by setting $a_{k}=\left|\left\langle T_{k} x, x\right\rangle\right|$ for all $(k=1,2, \cdots, n)$, we get

$$
\sum_{k=1}^{n}\left|\left\langle T_{k} x, x\right\rangle\right| \leq n^{1-\frac{1}{p}}\left(\sum_{k=1}^{n}\left|\left\langle T_{k} x, x\right\rangle\right|^{p}\right)^{\frac{1}{p}} .
$$

Taking the supremum over all unit vector $x \in \mathscr{H}$, one could get

$$
w_{R}\left(T_{1}, \cdots, T_{n}\right) \leq n^{1-\frac{1}{p}} w_{p}\left(T_{1}, \cdots, T_{n}\right) .
$$

Combining the inequalities (1.2) and (1.3) we get

$$
w_{\infty}\left(T_{1}, \cdots, T_{n}\right) \leq w_{p}\left(T_{1}, \cdots, T_{n}\right) \leq w_{R}\left(T_{1}, \cdots, T_{n}\right) \leq n^{1-\frac{1}{p}} w_{p}\left(T_{1}, \cdots, T_{n}\right) .
$$

More generally, in the power mean inequality

$$
\left(\frac{1}{n} \sum_{k=1}^{n} a_{k}^{p}\right)^{\frac{1}{p}} \leq\left(\frac{1}{n} \sum_{k=1}^{n} a_{k}^{q}\right)^{\frac{1}{q}}, \quad \forall p \leq q
$$


if one chooses $a_{k}=\left|\left\langle T_{k} x, x\right\rangle\right|$ for all $(k=1,2, \cdots, n)$, then we have

$$
\left(\frac{1}{n} \sum_{k=1}^{n}\left|\left\langle T_{k} x, x\right\rangle\right|^{p}\right)^{\frac{1}{p}} \leq\left(\frac{1}{n} \sum_{k=1}^{n}\left|\left\langle T_{k} x, x\right\rangle\right|^{q}\right)^{\frac{1}{q}} .
$$

Taking the supremum over all unit vector $x \in \mathscr{H}$, we get

$$
w_{p}\left(T_{1}, \cdots, T_{n}\right) \leq n^{\frac{1}{p}-\frac{1}{q}} w_{q}\left(T_{1}, \cdots, T_{n}\right), \quad \forall q \geq p \geq 1 .
$$

Indeed, one can refine (1.3) by applying the Jensen's inequality

$$
\left(\frac{1}{n} \sum_{k=1}^{n} a_{k}\right)^{p} \leq \frac{1}{n} \sum_{k=1}^{n} a_{k}^{p}-\frac{1}{n} \sum_{k=1}^{n}\left|a_{k}-\frac{1}{n} \sum_{j=1}^{n} a_{j}\right|^{p} \quad p \geq 2
$$

which obtained from more general result for superquadratic functions [1].

Thus, by setting $a_{k}=\left|\left\langle T_{k} x, x\right\rangle\right|$ in (1.6) we get

$$
\begin{aligned}
\left(\sum_{k=1}^{n}\left|\left\langle T_{k} x, x\right\rangle\right|\right)^{p} & \leq n^{p-1} \sum_{k=1}^{n}\left|\left\langle T_{k} x, x\right\rangle\right|^{p}-n^{p-1} \sum_{k=1}^{n}||\left\langle T_{k} x, x\right\rangle\left|-\frac{1}{n} \sum_{j=1}^{n}\right|\left\langle T_{j} x, x\right\rangle||^{p} \\
& \leq n^{p-1} \sum_{k=1}^{n}\left|\left\langle T_{k} x, x\right\rangle\right|^{p}-n^{p-1} \sum_{k=1}^{n}||\left\langle T_{k} x, x\right\rangle\left|-\frac{1}{n} \sup _{\|x\|=1} \sum_{j=1}^{n}\right|\left\langle T_{j} x, x\right\rangle||^{p}
\end{aligned}
$$

Taking the supremum again over all unit vector $x \in \mathscr{H}$, we get

$$
\begin{aligned}
& \sup _{\|x\|=1}\left(\sum_{k=1}^{n}\left|\left\langle T_{k} x, x\right\rangle\right|\right)^{p} \\
& \leq \sup _{\|x\|=1}\left\{n^{p-1} \sum_{k=1}^{n}\left|\left\langle T_{k} x, x\right\rangle\right|^{p}-n^{p-1} \sum_{k=1}^{n}||\left\langle T_{k} x, x\right\rangle\left|-\frac{1}{n} \sup _{\|x\|=1} \sum_{j=1}^{n}\right|\left\langle T_{j} x, x\right\rangle||^{p}\right\} \\
& \leq n^{p-1} \sup _{\|x\|=1} \sum_{k=1}^{n}\left|\left\langle T_{k} x, x\right\rangle\right|^{p}-\left.n^{p-1} \inf _{\|x\|=1} \sum_{k=1}^{n}||\left\langle T_{k} x, x\right\rangle\left|-\frac{1}{n} \sup _{\|x\|=1} \sum_{j=1}^{n}\right|\left\langle T_{j} x, x\right\rangle\right|^{p} \\
& =n^{p-1} w_{p}^{p}\left(T_{1}, \cdots, T_{n}\right)-n^{p-1} \inf _{\|x\|=1} \sum_{k=1}^{n}||\left\langle T_{k} x, x\right\rangle\left|-\frac{1}{n} w_{R}\left(T_{1}, \cdots, T_{n}\right)\right|^{p},
\end{aligned}
$$

which gives

$$
w_{R}^{p}\left(T_{1}, \cdots, T_{n}\right) \leq n^{p-1} w_{p}^{p}\left(T_{1}, \cdots, T_{n}\right)-n^{p-1} \inf _{\|x\|=1} \sum_{k=1}^{n}||\left\langle T_{k} x, x\right\rangle\left|-\frac{1}{n} w_{R}\left(T_{1}, \cdots, T_{n}\right)\right|^{p} .
$$

which refine the right hand side of (1.4). Clearly, all above mentioned inequalities generalize and refine some inequalities obtained in [20]. For recent inequalities, counterparts, refinements and other related properties concerning the generalized Euclidean operator radius the reader my refer to [5], [9], [12],[13], [21], [23] and[24].

\section{Bounds FOR THE GENERALIZED EuCLIDEAN OPERATOR RADIUS}

Lemma 1. We have

(1) The Power-Mean inequality

$$
a^{\alpha} b^{1-\alpha} \leq \alpha a+(1-\alpha) b \leq\left(\alpha a^{p}+(1-\alpha) b^{p}\right)^{\frac{1}{p}}
$$

for all $\alpha \in[0,1], a, b \geq 0$ and $p \geq 1$.

(2) The Power-Young inequality

$$
a b \leq \frac{a^{\alpha}}{\alpha}+\frac{b^{\beta}}{\beta} \leq\left(\frac{a^{p \alpha}}{\alpha}+\frac{b^{p \beta}}{\beta}\right)^{\frac{1}{p}}
$$


for all $a, b \geq 0$ and $\alpha, \beta>1$ with $\frac{1}{\alpha}+\frac{1}{\beta}=1$ and all $p \geq 1$.

Lemma 2. (The McCarty inequality). Let $A \in \mathscr{B}(\mathscr{H})^{+}$, then

$$
\langle A x, x\rangle^{p} \leq\left\langle A^{p} x, x\right\rangle, \quad p \geq 1
$$

for any unit vector $x \in \mathscr{H}$

Lemma 3. If $a, b>0$, and $p, q>1$ such that $\frac{1}{p}+\frac{1}{q}=1$, then for $m=1,2,3, \ldots$,

$$
\left(a^{\frac{1}{p}} b^{\frac{1}{q}}\right)^{m}+r_{0}^{m}\left(a^{\frac{m}{2}}-b^{\frac{m}{2}}\right)^{2} \leq\left(\frac{a^{r}}{p}+\frac{b^{r}}{q}\right)^{\frac{m}{r}}, r \geq 1,
$$

where $r_{0}=\min \left\{\frac{1}{p}, \frac{1}{q}\right\}$. In particular, if $p=q=2$, then

For $m=1$

$$
\left(a^{\frac{1}{2}} b^{\frac{1}{2}}\right)^{m}+\frac{1}{2^{m}}\left(a^{\frac{m}{2}}-b^{\frac{m}{2}}\right)^{2} \leq 2^{\frac{-m}{r}}\left(a^{r}+b^{r}\right)^{\frac{m}{r}} .
$$

$$
\left(a^{\frac{1}{2}} b^{\frac{1}{2}}\right)+\frac{1}{2}\left(a^{\frac{1}{2}}-b^{\frac{1}{2}}\right)^{2} \leq 2^{\frac{-1}{r}}\left(a^{r}+b^{r}\right)^{\frac{1}{r}} .
$$

In 1994, Furuta [11] proved the following generalization of Kato's inequality (1.3)

$$
\left|\left\langle T|T|^{\alpha+\beta-1} x, y\right\rangle\right|^{2} \leq\left\langle|T|^{2 \alpha} x, x\right\rangle\left\langle|T|^{2 \beta} y, y\right\rangle
$$

for any $x, y \in \mathscr{H}$ and $\alpha, \beta \in[0,1]$ with $\alpha+\beta \geq 1$.

The inequality (2.5) was generalized for any $\alpha, \beta \geq 0$ with $\alpha+\beta \geq 1$ by Dragomir in [8]. Indeed, as noted by Dragomir the condition $\alpha, \beta \in[0,1]$ was assumed by Furuta to fit with the Heinz-Kato inequality, which reads:

$$
|\langle T x, y\rangle| \leq\left\|A^{\alpha} x\right\|\left\|B^{1-\alpha} y\right\|
$$

for any $x, y \in \mathscr{H}$ and $\alpha \in[0,1]$ where $A$ and $B$ are positive operators such that $\|T x\| \leq\|A x\|$ and $\left\|T^{*} y\right\| \leq\|B y\|$ for any $x, y \in \mathscr{H}$.

In the same work [8], Dragomir provides a useful extension of Furuta's inequality, as follows:

$$
|\langle D C B A x, y\rangle|^{2} \leq\left\langle A^{*}|B|^{2} A x, x\right\rangle\left\langle D\left|C^{*}\right|^{2} D^{*} y, y\right\rangle
$$

for any $A, B, C, D \in \mathscr{B}(\mathscr{H})$ and any vectors $x, y \in \mathscr{H}$. The equality in (2.6) holds iff the vectors $B A x$ and $C^{*} D^{*} y$ are linearly dependent in $\mathscr{H}$. For other closely related version of Kato's inequality see [2], [14], [15], [18], [19] and [22].

2.1. Basic properties of the generalized Euclidean operator radius. Moslehian et al. [20], mention without proofs the following properties of the generalized Euclidean operator radius:

(1) $w_{p}\left(T_{1}, \cdots, T_{n}\right)=0$ if and only if $T_{k}=0$ for each $k=1, \cdots, n$.

(2) $w_{p}\left(\lambda T_{1}, \cdots, \lambda T_{n}\right)=|\lambda| w_{p}\left(T_{1}, \cdots, T_{n}\right)$.

(3) $w_{p}\left(A_{1}+B_{1}, \cdots, A_{n}+B_{n}\right) \leq w_{p}\left(A_{1}, \cdots, A_{n}\right)+w_{p}\left(B_{1}, \cdots, B_{n}\right)$.

(4) $w_{p}\left(X^{*} T_{1} X, \cdots, X^{*} T_{n} X\right)=\|X\| w_{p}\left(T_{1}, \cdots, T_{n}\right)$.

for every $T_{k}, A_{k}, B_{k}, X \in \mathscr{B}(\mathscr{H})(1 \leq k \leq n)$ and every scalar $\lambda \in \mathbb{C}$.

Despite of the authors in [20], mentioned the above basic properties of the generalized Euclidean operator radius, but it seems they missed some other important properties, rather than they left these properties without proof. Sometimes, it's nice to elaborate the proof of these elementary facts. Because of that we are going to give a proof of each property. Clearly, the first two properites follows from the definition of the generalized Euclidean operator radius. In what follows, and as the classical sense we have the following properties: 
Let $T_{1}, \cdots, T_{n}, U \in \mathscr{B}(\mathscr{H})$ such that $U$ is a unitary. Then, the following properties of the generalized Euclidean operator radius holds.

(1) The generalized Euclidean operator radius is weakly unitarily invariant i.e.,

$$
w_{p}\left(U^{*} T_{1} U, \cdots, U^{*} T_{n} U\right)=w_{p}\left(T_{1}, \cdots, T_{n}\right) .
$$

(2) $w_{p}\left(T_{1}, \cdots, T_{n}\right)=w_{p}\left(T_{1}^{*}, \cdots, T_{n}^{*}\right)$.

(3) $w_{p}\left(T_{1}^{*} T_{1}, \cdots, T_{n}^{*} T_{n}\right)=w_{p}\left(T_{1} T_{1}^{*}, \cdots, T_{n} T_{n}^{*}\right)$.

Proof. (1) The first property follows since

$$
\begin{aligned}
w_{p}\left(U_{1}^{*} T_{1} U_{1}, \cdots, U_{n}^{*} T_{n} U_{n}\right) & :=\sup _{\|x\|=1}\left(\sum_{i=1}^{n}\left|\left\langle U_{i}^{*} T_{i} U_{i} x, x\right\rangle\right|^{p}\right)^{1 / p} \\
& =\sup _{\|x\|=1}\left(\sum_{i=1}^{n}\left|\left\langle T_{i} U_{i} x, U_{i} x\right\rangle\right|^{p}\right)^{1 / p} \\
& =\sup _{\|y\|=1}\left(\sum_{i=1}^{n}\left|\left\langle T_{i} y, y\right\rangle\right|^{p}\right)^{1 / p} \\
& =w_{p}\left(T_{1}, \cdots, T_{n}\right) .
\end{aligned}
$$

(2) By the definition of the generalized Euclidean operator radius we have

$$
\begin{aligned}
w_{p}\left(T_{1}, \cdots, T_{n}\right):=\sup _{\|x\|=1}\left(\sum_{i=1}^{n}\left|\left\langle T_{i} x, x\right\rangle\right|^{p}\right)^{1 / p} & =\sup _{\|x\|=1}\left(\sum_{i=1}^{n}\left|\left\langle x, T_{i}^{*} x\right\rangle\right|^{p}\right)^{1 / p} \\
& =w_{p}\left(T_{1}^{*}, \cdots, T_{n}^{*}\right) .
\end{aligned}
$$

(3) Similarly, by definition we have

$$
\begin{aligned}
w_{p}\left(T_{1} T_{1}^{*}, \cdots, T_{n} T_{n}^{*}\right):=\sup _{\|x\|=1}\left(\sum_{i=1}^{n}\left|\left\langle T_{i} T_{i}^{*} x, x\right\rangle\right|^{p}\right)^{1 / p} & =\sup _{\|x\|=1}\left(\sum_{i=1}^{n}\left|\left\langle x, T_{i} T_{i}^{*} x\right\rangle\right|^{p}\right)^{1 / p} \\
& =w_{p}\left(T_{1}^{*} T_{1}, \cdots, T_{n}^{*} T_{n}\right) .
\end{aligned}
$$

(4) Finally, employing the classical Minlowski inequality, i.e., we get

$$
\begin{aligned}
w_{p}\left(A_{1}+B_{1}, \cdots, A_{n}+B_{n}\right) & =\left(\sum_{i=}^{n}\left|\left\langle\left(A_{i}+B_{i}\right) x, x\right\rangle\right|^{p}\right)^{\frac{1}{p}} \\
& =\left(\sum_{i=}^{n}\left(\left|\left\langle A_{i} x, x\right\rangle\right|+\left|\left\langle B_{i} x, x\right\rangle\right|\right)^{p}\right)^{\frac{1}{p}} \\
& \leq\left(\sum_{i=}^{n}\left|\left\langle A_{i} x, x\right\rangle\right|^{p}\right)^{\frac{1}{p}}+\left(\sum_{i=}^{n}\left|\left\langle B_{i} x, x\right\rangle\right|^{p}\right)^{\frac{1}{p}} \\
& =w_{p}\left(A_{1}, \cdots, A_{n}\right)+w_{p}\left(B_{1}, \cdots, B_{n}\right) .
\end{aligned}
$$

which proves the last property. 
It remains to prove $w_{p}\left(X^{*} T_{1} X, \cdots, X^{*} T_{n} X\right)=\|X\| w_{p}\left(T_{1}, \cdots, T_{n}\right)$. Form the definition of the generalized Euclidean operator radius, we have

$$
\begin{aligned}
w_{p}\left(X^{*} T_{1} X, \cdots, X^{*} T_{n} X\right) & :=\sup _{\|x\|=1}\left(\sum_{i=1}^{n}\left|\left\langle X^{*} T_{i} X x, x\right\rangle\right|^{p}\right)^{1 / p} \\
& =\sup _{\|x\|=1}\left(\sum_{i=1}^{n}\left|\left\langle T_{i} X x, X x\right\rangle\right|^{p}\right)^{1 / p} \\
& \leq \sup _{\|x\|=1}\left(\sum_{i=1}^{n}\|X\|^{2}\left|\left\langle T_{i} x, x\right\rangle\right|^{p}\right)^{1 / p} \\
& =\|X\|^{2} \sup _{\|x\|=1}\left(\sum_{i=1}^{n}\left|\left\langle T_{i} x, x\right\rangle\right|^{p}\right)^{1 / p} \\
& =\|X\|^{2} w_{p}\left(T_{1}^{*}, \cdots, T_{n}^{*}\right) .
\end{aligned}
$$

as required.

Proposition 1. Let $T_{1}, \cdots, T_{n} \in \mathscr{B}(\mathscr{H})$ and $f, g$ are nonnegative continuous functions defined on $[0, \infty)$ satisfying that $f(t) g(t)=t(t \geq 0)$. Then,

$$
w_{r}^{r}\left(T_{1}, \cdots, T_{n}\right) \leq w_{p}\left(f^{r}\left(\left|T_{1}\right|\right), \cdots, f^{r}\left(\left|T_{n}\right|\right)\right) w_{q}\left(g^{r}\left(\left|T_{1}^{*}\right|\right), \cdots, g^{r}\left(\left|T_{n}^{*}\right|\right)\right)
$$

for all $r \geq 2$ and $p, q>1$ with $\frac{1}{p}+\frac{1}{q}=1$.

Proof.

$$
\begin{aligned}
\left|\left\langle T_{i} x, x\right\rangle\right|^{r} & \leq\left\|f\left(\left|T_{i}\right|\right) x\right\|^{r}\left\|g\left(\left|T_{i}^{*}\right|\right) x\right\|^{r} \\
& =\left\langle f^{2}\left(\left|T_{i}\right|\right) x, x\right\rangle^{\frac{r}{2}}\left\langle g^{2}\left(\left|T_{i}^{*}\right|\right) x, x\right\rangle^{\frac{r}{2}} \\
& \leq\left\langle f^{r}\left(\left|T_{i}\right|\right) x, x\right\rangle\left\langle g^{r}\left(\left|T_{i}^{*}\right|\right) x, x\right\rangle \quad \text { (by the McCarthy inequality) }
\end{aligned}
$$

Taking the sum over all $i$ from 1 to $n$ we get

$$
\begin{aligned}
\sum_{i=1}^{n}\left|\left\langle T_{i} x, x\right\rangle\right|^{r} & \leq \sum_{I=1}^{n}\left\langle f^{r}\left(\left|T_{i}\right|\right) x, x\right\rangle\left\langle g^{r}\left(\left|T_{i}^{*}\right|\right) x, x\right\rangle \\
& \leq\left(\sum_{I=1}^{n}\left\langle f^{r}\left(\left|T_{i}\right|\right) x, x\right\rangle^{p}\right)^{\frac{1}{p}}\left(\sum_{I=1}^{n}\left\langle g^{r}\left(\left|T_{i}^{*}\right|\right) x, x\right\rangle^{q}\right)^{\frac{1}{q}} \quad \text { (by the Hölder inequality) }
\end{aligned}
$$

Taking the supremum over all vectors $x \in \mathscr{H}$ such that $\|x\|=1$, we get the desired result.

Corollary 1. Let $T_{1}, \cdots, T_{n} \in \mathscr{B}(\mathscr{H})$ and $f, g$ are nonnegative continuous functions defined on $[0, \infty)$ satisfying that $f(t) g(t)=t(t \geq 0)$. Then,

$$
w_{r}^{r}\left(T_{1}, \cdots, T_{n}\right) \leq w_{\mathrm{e}}\left(f^{r}\left(\left|T_{1}\right|\right), \cdots, f^{r}\left(\left|T_{n}\right|\right)\right) w_{\mathrm{e}}\left(g^{r}\left(\left|T_{1}^{*}\right|\right), \cdots, g^{r}\left(\left|T_{n}^{*}\right|\right)\right)
$$

for all $r \geq 2$.

Proof. Setting $p=q=2$ in (2.7).

Proposition 2. Let $A_{i}, B_{i}, C_{i}, D_{i} \in \mathscr{B}(\mathscr{H})(i=1, \cdots, n)$. Then,

$$
\begin{aligned}
& w_{e}^{2}\left(D_{1} C_{1} B_{1} A_{1}, \cdots, D_{n} C_{n} B_{n} A_{n}\right) \\
& \quad \leq w_{p}\left(A_{1}^{*}\left|B_{1}\right|^{2} A_{1}, \cdots, A_{n}^{*}\left|B_{n}\right|^{2} A_{n}\right) w_{q}\left(D_{1}\left|C_{1}^{*}\right|^{2} D_{1}^{*}, \cdots, D_{n}\left|C_{n}^{*}\right|^{2} D_{n}^{*}\right)
\end{aligned}
$$


Proof. Let $y=x$ in (2.6), we get

$$
\begin{aligned}
& \sup _{\|x\|=1} \sum_{i=1}^{n}\left|\left\langle D_{i} C_{i} B_{i} A_{i} x, x\right\rangle\right|^{2} \\
& \leq \sup _{\|x\|=1} \sum_{i=1}^{n}\left\langle A_{i}^{*}\left|B_{i}\right|^{2} A_{i} x, x\right\rangle\left\langle D_{i}\left|C_{i}^{*}\right|^{2} D_{i}^{*} x, x\right\rangle \\
& \leq \sup _{\|x\|=1}\left(\sum_{i=1}^{n}\left\langle A_{i}^{*}\left|B_{i}\right|^{2} A_{i} x, x\right\rangle^{p}\right)^{\frac{1}{p}} \sup _{\|x\|=1}\left(\sum_{i=1}^{n}\left\langle D_{i}\left|C_{i}^{*}\right|^{2} D_{i}^{*} x, x\right\rangle^{q}\right)^{\frac{1}{q}} \\
& =w_{p}\left(A_{1}^{*}\left|B_{1}\right|^{2} A_{1}, \cdots, A_{n}^{*}\left|B_{n}\right|^{2} A_{n}\right) w_{q}\left(D_{1}\left|C_{1}^{*}\right|^{2} D_{1}^{*}, \cdots, D_{n}\left|C_{n}^{*}\right|^{2} D_{n}^{*}\right),
\end{aligned}
$$

which is gives the desired result.

Corollary 2. Let $B_{i} \in \mathscr{B}(\mathscr{H})(i=1, \cdots, n)$. Then,

$$
w_{e}^{2}\left(B_{1}^{2}, \cdots, B_{n}^{2}\right) \leq w_{p}\left(\left|B_{1}\right|^{2}, \cdots,\left|B_{n}\right|^{2}\right) w_{q}\left(\left|B_{1}\right|^{2}, \cdots,\left|B_{n}\right|^{2}\right)
$$

for all $p, q>1$ such that $\frac{1}{p}+\frac{1}{q}=1$. In particular, for $p=q=2$ we have

$$
w_{\mathrm{e}}\left(B_{1}^{2}, \cdots, B_{n}^{2}\right) \leq w_{\mathrm{e}}\left(\left|B_{1}\right|^{2}, \cdots,\left|B_{n}\right|^{2}\right)
$$

Proof. Setting $A_{i}=U_{i}, D_{i}=U_{i}^{*}\left(U_{i}\right.$ are unitaries for all $\left.i=1, \cdots, n\right)$ and $C_{i}=B_{i}$ in the previous result. Then

$$
w_{\mathrm{e}}^{2}\left(U_{1}^{*} B_{1}^{2} U_{1}, \cdots, U_{n}^{*} B_{n}^{2} U_{n}\right) \leq w_{p}\left(U_{1}^{*}\left|B_{1}\right|^{2} U_{1}, \cdots, U_{n}^{*}\left|B_{n}\right|^{2} U_{n}\right) w_{q}\left(U_{1}^{*}\left|B_{1}^{*}\right|^{2} U_{1}, \cdots, U_{n}^{*}\left|B_{n}^{*}\right|^{2} U_{n}\right) .
$$

Since $w_{p}(\cdot)$ is weakly unitarily invariant and

$$
w_{q}\left(\left|B_{1}^{*}\right|^{2}, \cdots,\left|B_{n}^{*}\right|^{2}\right)=w_{q}\left(\left|B_{1}\right|^{2}, \cdots,\left|B_{n}\right|^{2}\right) .
$$

Thus, the desired result is obtained.

Corollary 3. Let $T_{i} \in \mathscr{B}(\mathscr{H})(i=1, \cdots, n), \alpha, \beta \geq 0$ such that $\alpha+\beta \geq 1$. Then,

$$
w_{\mathrm{e}}^{2}\left(T_{1}\left|T_{1}\right|^{\alpha+\beta-1}, \cdots, T_{n}\left|T_{n}\right|^{\alpha+\beta-1}\right) \leq w_{p}\left(\left|T_{1}\right|^{2 \alpha}, \cdots,\left|T_{n}\right|^{2 \alpha}\right) w_{q}\left(\left|T_{1}^{*}\right|^{2 \beta}, \cdots,\left|T_{n}^{*}\right|^{2 \beta}\right)
$$

for all $p, q>1$ such that $\frac{1}{p}+\frac{1}{q}=1$.

Proof. Let $U_{i}$ be unitaries for all $i=1, \cdots, n$, setting $D_{i}=U_{i}, B=1_{\mathscr{H}}, C=\left|T_{i}\right|^{\beta}$ and $A_{i}=\left|T_{i}\right|^{\alpha}$ for all $\alpha, \beta \geq 0$ such that $\alpha+\beta \geq 1$ in (2.9), then we have

$$
D_{i} C_{i} B_{i} A_{i}=U_{i}\left|T_{i}\right|^{\beta}\left|T_{i}\right|^{\alpha}=U_{i}\left|T_{i}\right|\left|T_{i}\right|^{\alpha+\beta-1}=T_{i}\left|T_{i}\right|^{\alpha+\beta-1},
$$

also, we have $A_{i}^{*}\left|B_{i}\right|^{2} A_{i}=\left|T_{i}\right|^{2 \alpha}$ and $D_{i}\left|C_{i}^{*}\right|^{2} D_{i}^{*}=U_{i}\left|T_{i}\right|^{2 \beta} U_{i}^{*}=\left|T_{i}\right|^{2 \beta}$ for all $i=1, \cdots, n$.

Corollary 4. Let $T_{i} \in \mathscr{B}(\mathscr{H})(i=1, \cdots, n)$. Then,

$$
w_{\mathrm{e}}^{2}\left(T_{1}, \cdots, T_{n}\right) \leq w_{p}\left(\left|T_{1}\right|, \cdots,\left|T_{n}\right|\right) w_{q}\left(\left|T_{1}^{*}\right|, \cdots,\left|T_{n}^{*}\right|\right)
$$

for all $p, q>1$ such that $\frac{1}{p}+\frac{1}{q}=1$.

Proof. Setting $\alpha=\beta=\frac{1}{2}$ in (2.12).

Corollary 5. Let $T_{i} \in \mathscr{B}(\mathscr{H})(i=1, \cdots, n), \alpha, \beta \geq 0$ such that $\alpha+\beta \geq 1$. Then,

$$
w_{\mathrm{e}}^{2}\left(T_{1}\left|T_{1}\right|, \cdots, T_{n}\left|T_{n}\right|\right) \leq w_{p}\left(\left|T_{1}\right|^{2}, \cdots,\left|T_{n}\right|^{2}\right) w_{q}\left(\left|T_{1}\right|^{2}, \cdots,\left|T_{n}\right|^{2}\right)
$$

for all $p, q>1$ such that $\frac{1}{p}+\frac{1}{q}=1$. In particular, for $p=q=2$ we have

$$
w_{\mathrm{e}}^{2}\left(T_{1}\left|T_{1}\right|, \cdots, T_{n}\left|T_{n}\right|\right) \leq w_{\mathrm{e}}^{2}\left(\left|T_{1}\right|^{2}, \cdots,\left|T_{n}\right|^{2}\right)
$$

Proof. Setting $\alpha=\beta=1$ in (2.12) and use the properties of $w_{p}(\cdot)$. 


\subsection{Inequalities for the generalized Euclidean operator radius.}

Theorem 1. Let $A_{i}, B_{i}, C_{i}, D_{i} \in \mathscr{B}(\mathscr{H})(i=1, \cdots, n)$. Then,

$$
\begin{aligned}
w_{R}\left(D_{1} C_{1} B_{1} A_{1}, \cdots, D_{n} C_{n} B_{n} A_{n}\right) \leq & \| \sum_{i=1}^{n} \frac{1}{2 p}\left[\left(A_{i}^{*}\left|B_{i}\right|^{2} A_{i}\right)^{(1-\gamma) p r}+\left(A_{i}^{*}\left|B_{i}\right|^{2} A_{i}\right)^{\gamma p r}\right] \\
& +\frac{1}{2 q}\left[\left(D_{i}\left|C_{i}^{*}\right|^{2} D_{i}^{*}\right)^{\gamma q r}+\left(D_{i}\left|C_{i}^{*}\right|^{2} D_{i}^{*}\right)^{(1-\gamma) q r}\right] \|
\end{aligned}
$$

for all $r \geq 1, p, q>1$ and $\gamma \in[0,1]$ such that $p \gamma \geq 2$ and $\frac{1}{p}+\frac{1}{q}=1$.

Proof. Let $y=x$ in (2.6), then we have

$$
\begin{aligned}
& \sum_{i=1}^{n}\left|\left\langle D_{i} C_{i} B_{i} A_{i} x, x\right\rangle\right| \\
& =\sum_{i=1}^{n}\left\langle A_{i}^{*}\left|B_{i}\right|^{2} A_{i} x, x\right\rangle^{\frac{1}{2}}\left\langle D_{i}\left|C_{i}^{*}\right|^{2} D_{i}^{*} x, x\right\rangle^{\frac{1}{2}} \\
& \leq \frac{1}{2} \sum_{i=1}^{n}\left[\left\langle A_{i}^{*}\left|B_{i}\right|^{2} A_{i} x, x\right\rangle^{1-\gamma}\left\langle D_{i}\left|C_{i}^{*}\right|^{2} D_{i}^{*} x, x\right\rangle^{\gamma} \quad \text { (since } \sqrt{a b} \leq \frac{a^{\gamma} b^{1-\gamma}+a^{1-\gamma} b^{\gamma}}{2}\right) \\
& \left.+\left\langle A_{i}^{*}\left|B_{i}\right|^{2} A_{i} x, x\right\rangle^{\gamma}\left\langle D_{i}\left|C_{i}^{*}\right|^{2} D_{i}^{*} x, x\right\rangle^{1-\gamma}\right] \quad \text { (by Hölder inequality) } \\
& \leq \frac{1}{2} \sum_{i=1}^{n}\left\{\left(\left\langle A_{i}^{*}\left|B_{i}\right|^{2} A_{i} x, x\right\rangle^{(1-\gamma) p}+\left\langle A_{i}^{*}\left|B_{i}\right|^{2} A_{i} x, x\right\rangle^{\gamma p}\right)^{\frac{1}{p}}\right. \\
& \left.\quad \times\left(\left\langle D_{i}\left|C_{i}^{*}\right|^{2} D_{i}^{*} x, x\right\rangle^{\gamma q}+\left\langle D_{i}\left|C_{i}^{*}\right|^{2} D_{i}^{*} x, x\right\rangle^{(1-\gamma) q}\right)^{\frac{1}{q}}\right\} \\
& \leq \frac{1}{2 p} \sum_{i=1}^{n}\left(\left\langle\left(A_{i}^{*}\left|B_{i}\right|^{2} A_{i}\right)^{(1-\gamma) p} x, x\right\rangle+\left\langle\left(A_{i}^{*}\left|B_{i}\right|^{2} A_{i}\right)^{\gamma p} x, x\right\rangle\right) \\
& \quad+\frac{1}{2 q} \sum_{i=1}^{n}\left(\left\langle\left(D_{i}\left|C_{i}^{*}\right|^{2} D_{i}^{*}\right)^{\gamma q} x, x\right\rangle+\left\langle\left(D_{i}\left|C_{i}^{*}\right|^{2} D_{i}^{*}\right)^{(1-\gamma) q} x, x\right\rangle\right) \\
& \left.\left.\quad+\frac{1}{2 q}\left[\left(D_{i}\left|C_{i}^{*}\right|^{2} D_{i}^{*}\right)^{\gamma q}+\left(D_{i}\left|C_{i}^{*}\right|^{2} D_{i}^{*}\right)^{(1-\gamma) q}\right]\right\} x, x\right\rangle .
\end{aligned}
$$

Taking the supremum over all unit vector $x \in \mathscr{H}$ we get the required result.

Corollary 6. Let $A_{i}, B_{i}, C_{i}, D_{i} \in \mathscr{B}(\mathscr{H})(i=1, \cdots, n)$. Then,

$$
\begin{aligned}
& w_{R}\left(D_{1} C_{1} B_{1} A_{1}, \cdots, D_{n} C_{n} B_{n} A_{n}\right) \\
\leq & \left\|\sum_{i=1}^{n}\left\{\frac{1}{4}\left[\left(A_{i}^{*}\left|B_{i}\right|^{2} A_{i}\right)^{2(1-\gamma)}+\left(A_{i}^{*}\left|B_{i}\right|^{2} A_{i}\right)^{2 \gamma}\right]+\left[\left(D_{i}\left|C_{i}^{*}\right|^{2} D_{i}^{*}\right)^{2 \gamma}+\left(D_{i}\left|C_{i}^{*}\right|^{2} D_{i}^{*}\right)^{2(1-\gamma)}\right]\right\}\right\|
\end{aligned}
$$

for all $\gamma \in[0,1]$

Proof. Setting $p=q=2$ and $r=1$ in (2.16). 
Corollary 7. $\operatorname{Let} T_{i} \in \mathscr{B}(\mathscr{H})(i=1, \cdots, n)$. Then,

$$
\begin{aligned}
w_{R}\left(T_{1}\left|T_{1}\right|^{\alpha+\beta-1}, \cdots,\right. & \left.T_{n}\left|T_{n}\right|^{\alpha+\beta-1}\right) \\
& \leq\left\|\sum_{i=1}^{n}\left\{\frac{1}{2 p}\left[\left|T_{i}\right|^{2 \alpha(1-\gamma) p r}+\left|T_{i}\right|^{2 \alpha \gamma p r}\right]+\frac{1}{2 q}\left[\left|T_{i}^{*}\right|^{2 \beta \gamma q r}+\left|T_{i}^{*}\right|^{2 \beta(1-\gamma) q r}\right]\right\}\right\|
\end{aligned}
$$

for all $r \geq 1, p, q>1$ and $\gamma \in[0,1]$ such that $p \gamma \geq 2$ and $\frac{1}{p}+\frac{1}{q}=1$.

In particular, we have

$$
\begin{aligned}
& w_{R}\left(T_{1}\left|T_{1}\right|^{\alpha+\beta-1}, \cdots, T_{n}\left|T_{n}\right|^{\alpha+\beta-1}\right) \\
& \quad \leq \frac{1}{4}\left\|\sum_{i=1}^{n}\left\{\left[\left|T_{i}\right|^{4 \alpha(1-\gamma) r}+\left|T_{i}\right|^{4 \alpha \gamma r}\right]+\left[\left|T_{i}^{*}\right|^{4 \beta \gamma r}+\left|T_{i}^{*}\right|^{4 \beta(1-\gamma) r}\right]\right\}\right\|
\end{aligned}
$$

Proof. The proof is similar to the inequality (2.12) by employing (2.16).

Remark 1. Setting $\gamma=1, \alpha=\beta=\frac{1}{2}$ and $r=2$ in (2.19), so that we have

$$
w_{R}\left(T_{1}, \cdots, T_{n}\right) \leq \frac{1}{4}\left\|\sum_{i=1}^{n}\left\{\left[1_{\mathscr{H}}+\left|T_{i}\right|^{4}\right]+\left[\left|T_{i}^{*}\right|^{4}+1 \mathscr{H}\right]\right\}\right\|
$$

Corollary 8. Let $T_{i} \in \mathscr{B}(\mathscr{H})(i=1, \cdots, n)$. Then,

$$
w_{R}\left(T_{1}\left|T_{1}\right|^{\alpha+\beta-1}, \cdots, T_{n}\left|T_{n}\right|^{\alpha+\beta-1}\right) \leq \frac{1}{4}\left\|\sum_{i=1}^{n}\left[\left|T_{i}\right|^{\frac{8}{3} \alpha}+\left|T_{i}\right|^{\frac{4}{3} \alpha}+\left|T_{i}^{*}\right|^{\frac{8}{3} \beta}+\left|T_{i}^{*}\right|^{\frac{4}{3} \beta}\right]\right\|
$$

Proof. Setting $p=q=2, r=1$ and $\gamma=\frac{1}{3}$ in (2.19).

Corollary 9. $\operatorname{Let} T_{i} \in \mathscr{B}(\mathscr{H})(i=1, \cdots, n)$. Then,

$$
w_{R}\left(T_{1}\left|T_{1}\right|^{\frac{1}{2}}, \cdots, T_{n}\left|T_{n}\right|^{\frac{1}{2}}\right) \leq \frac{1}{4}\left\|\sum_{i=1}^{n}\left(\left|T_{i}\right|^{2}+\left|T_{i}^{*}\right|^{2}+\left|T_{i}\right|+\left|T_{i}^{*}\right|\right)\right\|
$$

Proof. Setting $\alpha=\beta=\frac{3}{4}$ in (2.21).

Theorem 2. Let $A_{i}, B_{i}, C_{i}, D_{i} \in \mathscr{B}(\mathscr{H})(i=1, \cdots, n)$. Then,

$$
\begin{aligned}
& w_{p}^{p}\left(D_{1} C_{1} B_{1} A_{1}, \cdots, D_{n} C_{n} B_{n} A_{n}\right) \\
& \quad \leq \frac{1}{2}\left\|\sum_{i=1}^{n}\left[(1-\gamma) A_{i}^{*}\left|B_{i}\right|^{2} A_{i}+\gamma D_{i}\left|C_{i}^{*}\right|^{2} D_{i}^{*}\right]^{p}+\left[\gamma A_{i}^{*}\left|B_{i}\right|^{2} A_{i}+(1-\gamma) D_{i}\left|C_{i}^{*}\right|^{2} D_{i}^{*}\right]^{p}\right\|
\end{aligned}
$$

for all $\gamma \in[0,1]$ and $p \geq 1$. In particular, we have

$$
w_{p}^{p}\left(D_{1} C_{1} B_{1} A_{1}, \cdots, D_{n} C_{n} B_{n} A_{n}\right) \leq \frac{1}{2^{p}}\left\|\sum_{i=1}^{n}\left(A_{i}^{*}\left|B_{i}\right|^{2} A_{i}+D_{i}\left|C_{i}^{*}\right|^{2} D_{i}^{*}\right)^{p}\right\|
$$


Proof. Let $y=x$ in (2.6), then we have

$$
\begin{aligned}
& w_{p}^{p}\left(D_{1} C_{1} B_{1} A_{1}, \cdots, D_{n} C_{n} B_{n} A_{n}\right) \\
& =\sum_{i=1}^{n}\left|\left\langle D_{i} C_{i} B_{i} A_{i} x, x\right\rangle\right|^{p} \\
& =\sum_{i=1}^{n}\left(\left\langle A_{i}^{*}\left|B_{i}\right|^{2} A_{i} x, x\right\rangle^{\frac{1}{2}}\left\langle D_{i}\left|C_{i}^{*}\right|^{2} D_{i}^{*} x, x\right\rangle^{\frac{1}{2}}\right)^{p} \\
& \leq \frac{1}{2^{p}} \sum_{i=1}^{n}\left[\left(\left\langle A_{i}^{*}\left|B_{i}\right|^{2} A_{i} x, x\right\rangle^{(1-\gamma)}\left\langle D_{i}\left|C_{i}^{*}\right|^{2} D_{i}^{*} x, x\right\rangle^{\gamma}\right) \quad \quad\left(\text { since } \sqrt{a b} \leq \frac{a^{\gamma} b^{1-\gamma}+a^{1-\gamma} b^{\gamma}}{2}\right)\right. \\
& \left.+\left(\left\langle A_{i}^{*}\left|B_{i}\right|^{2} A_{i} x, x\right\rangle^{\gamma}\left\langle D_{i}\left|C_{i}^{*}\right|^{2} D_{i}^{*} x, x\right\rangle^{(1-\gamma)}\right)\right]^{p} \\
& \leq \frac{1}{2} \sum_{i=1}^{n}\left[\left((1-\gamma)\left\langle A_{i}^{*}\left|B_{i}\right|^{2} A_{i} x, x\right\rangle+\gamma\left\langle D_{i}\left|C_{i}^{*}\right|^{2} D_{i}^{*} x, x\right\rangle\right)^{p}\right. \\
& \left.+\left(\gamma\left\langle A_{i}^{*}\left|B_{i}\right|^{2} A_{i} x, x\right\rangle+(1-\gamma)\left\langle D_{i}\left|C_{i}^{*}\right|^{2} D_{i}^{*} x, x\right\rangle\right)^{p}\right] \\
& =\frac{1}{2} \sum_{i=1}^{n}\left\langle\left[(1-\gamma) A_{i}^{*}\left|B_{i}\right|^{2} A_{i}+\gamma D_{i}\left|C_{i}^{*}\right|^{2} D_{i}^{*}\right] x, x\right\rangle^{p} \\
& +\frac{1}{2} \sum_{i=1}^{n}\left\langle\left[\gamma A_{i}^{*}\left|B_{i}\right|^{2} A_{i}+(1-\gamma) D_{i}\left|C_{i}^{*}\right|^{2} D_{i}^{*}\right] x, x\right\rangle^{p} \\
& \leq \frac{1}{2} \sum_{i=1}^{n}\left\langle\left[(1-\gamma) A_{i}^{*}\left|B_{i}\right|^{2} A_{i}+\gamma D_{i}\left|C_{i}^{*}\right|^{2} D_{i}^{*}\right]^{p} x, x\right\rangle \quad \text { (by McCarthy inequality) } \\
& +\frac{1}{2} \sum_{i=1}^{n}\left\langle\left[\gamma A_{i}^{*}\left|B_{i}\right|^{2} A_{i}+(1-\gamma) D_{i}\left|C_{i}^{*}\right|^{2} D_{i}^{*}\right]^{p} x, x\right\rangle \\
& =\frac{1}{2}\left\langle\sum_{i=1}^{n}\left\{\left[(1-\gamma) A_{i}^{*}\left|B_{i}\right|^{2} A_{i}+\gamma D_{i}\left|C_{i}^{*}\right|^{2} D_{i}^{*}\right]^{p}\right\}\right. \text {, } \\
& \left.\left.+\left[\gamma A_{i}^{*}\left|B_{i}\right|^{2} A_{i}+(1-\gamma) D_{i}\left|C_{i}^{*}\right|^{2} D_{i}^{*}\right]^{p} x, x\right\rangle\right] .
\end{aligned}
$$

Taking the supremum over all unit vector $x \in \mathscr{H}$ we get the required result. The particular case is obtained by setting $\gamma=\frac{1}{2}$ in $(2.22)$.

Corollary 10. Let $T_{i} \in \mathscr{B}(\mathscr{H})(i=1, \cdots, n)$. Then, for all $\alpha, \beta \geq 0$ such that $\alpha+\beta \geq 1$ we have

$$
w_{p}^{p}\left(T_{1}\left|T_{1}\right|^{\alpha+\beta-1}, \cdots, T_{n}\left|T_{n}\right|^{\alpha+\beta-1}\right) \leq \frac{1}{2^{p}}\left\|\sum_{i=1}^{n}\left(\left|T_{i}\right|^{2 \alpha}+\left|T_{i}^{*}\right|^{2 \beta}\right)^{p}\right\|
$$

for all $p \geq 1$.

Proof. Let $U_{i}$ be unitaries for all $i=1, \cdots, n$, setting $D_{i}=U_{i}, B=1_{\mathscr{H}}, C=\left|T_{i}\right|^{\beta}$ and $A_{i}=\left|T_{i}\right|^{\alpha}$ for all $\alpha, \beta \geq 0$ such that $\alpha+\beta \geq 1$ in (2.23), then we have

$$
D_{i} C_{i} B_{i} A_{i}=U_{i}\left|T_{i}\right|^{\beta}\left|T_{i}\right|^{\alpha}=U_{i}\left|T_{i}\right|\left|T_{i}\right|^{\alpha+\beta-1}=T_{i}\left|T_{i}\right|^{\alpha+\beta-1},
$$

also, we have $A_{i}^{*}\left|B_{i}\right|^{2} A_{i}=\left|T_{i}\right|^{2 \alpha}$ and $D_{i}\left|C_{i}^{*}\right|^{2} D_{i}^{*}=U_{i}\left|T_{i}\right|^{2 \beta} U_{i}^{*}=\left|T_{i}\right|^{2 \beta}$ for all $i=1, \cdots, n$.

Corollary 11. Let $T_{i} \in \mathscr{B}(\mathscr{H})(i=1, \cdots, n)$. Then, we have

$$
w_{p}^{p}\left(T_{1}\left|T_{1}\right|, \cdots, T_{n}\left|T_{n}\right|\right) \leq \frac{1}{2^{p}}\left\|\sum_{i=1}^{n}\left(\left|T_{i}\right|^{2}+\left|T_{i}^{*}\right|^{2}\right)^{p}\right\|
$$

Proof. Setting $\alpha=\beta=\frac{1}{2}$ in (2.24). 
Theorem 3. Let $A_{i}, B_{i}, C_{i}, D_{i} \in \mathscr{B}(\mathscr{H})(i=1, \cdots, n)$. Then, for $m \in \mathbb{N}$ and $r, p \geq m \geq 1$,

$$
\begin{aligned}
& w_{p}^{p}\left(D_{1} C_{1} B_{1} A_{1}, \cdots, D_{n} C_{n} B_{n} A_{n}\right) \\
& \quad \leq \frac{n^{1-\frac{m}{r}}}{2^{\frac{m}{r}}}\left\|\sum_{i=1}^{n}\left(\left(A_{i}^{*}\left|B_{i}\right|^{2} A_{i}\right)^{\frac{r p}{m}}+\left(D_{i}\left|C_{i}^{*}\right|^{2} D_{i}^{*}\right)^{\frac{r p}{m}}\right)\right\|^{\frac{m}{r}}-\inf _{\|x\|=1} \xi_{p, m}(x),
\end{aligned}
$$

where

$$
\xi_{p, m}(x):=2^{-m} \sum_{i=1}^{n}\left(\left\langle\left(A_{i}^{*}\left|B_{i}\right|^{2} A_{i}\right)^{\frac{p}{m}} x, x\right\rangle^{\frac{m}{2}}-\left\langle\left(D_{i}\left|C_{i}^{*}\right|^{2} D_{i}^{*}\right)^{\frac{p}{m}} x, x\right\rangle^{\frac{m}{2}}\right)^{2} .
$$

Proof. Let $x \in \mathscr{H}$ be a unit vector. Let $y=x$ in (2.6) then we have

$$
\begin{aligned}
& \sum_{i=1}^{n}\left|\left\langle D_{i} C_{i} B_{i} A_{i} x, x\right\rangle\right|^{p} \\
& =\sum_{i=1}^{n}\left\langle A_{i}^{*}\left|B_{i}\right|^{2} A_{i} x, x\right\rangle^{\frac{p}{2}}\left\langle D_{i}\left|C_{i}^{*}\right|^{2} D_{i}^{*} x, x\right\rangle^{\frac{p}{2}} \\
& =\sum_{i=1}^{n}\left(\left\langle A_{i}^{*}\left|B_{i}\right|^{2} A_{i} x, x\right\rangle^{\frac{p}{2 m}}\left\langle D_{i}\left|C_{i}^{*}\right|^{2} D_{i}^{*} x, x\right\rangle^{\frac{p}{2 m}}\right)^{m} \\
& \leq \sum_{i=1}^{n}\left(\left\langle\left(A_{i}^{*}\left|B_{i}\right|^{2} A_{i}\right)^{\frac{p}{m}} x, x\right\rangle^{\frac{1}{2}}\left\langle\left(D_{i}\left|C_{i}^{*}\right|^{2} D_{i}^{*}\right)^{\frac{p}{m}} x, x\right\rangle^{\frac{1}{2}}\right)^{m} \quad \text { (by McCarthy inequality) } \\
& \begin{aligned}
\leq \sum_{i=1}^{n} & \left(\frac{1}{2}\left\langle\left(A_{i}^{*}\left|B_{i}\right|^{2} A_{i}\right)^{\frac{p}{m}} x, x\right\rangle^{r}+\left\langle\left(D_{i}\left|C_{i}^{*}\right|^{2} D_{i}^{*}\right)^{\frac{p}{m}} x, x\right\rangle^{r}\right)^{\frac{m}{r}} \\
& \quad-2^{-m} \sum_{i=1}^{n}\left(\left\langle\left(A_{i}^{*}\left|B_{i}\right|^{2} A_{i}\right)^{\frac{p}{m}} x, x\right\rangle^{\frac{m}{2}}-\left\langle\left(D_{i}\left|C_{i}^{*}\right|^{2} D_{i}^{*}\right)^{\frac{p}{m}} x, x\right\rangle^{\frac{m}{2}}\right)^{2}
\end{aligned} \\
& \begin{aligned}
\leq & \sum_{i=1}^{n}\left(\frac{1}{2}\left\langle\left(A_{i}^{*}\left|B_{i}\right|^{2} A_{i}\right)^{\frac{r p}{m}} x, x\right\rangle+\left\langle\left(D_{i}\left|C_{i}^{*}\right|^{2} D_{i}^{*}\right)^{\frac{r p}{m}} x, x\right\rangle\right)^{\frac{m}{r}} \\
& \quad-2^{-m} \sum_{i=1}^{n}\left(\left\langle\left(A_{i}^{*}\left|B_{i}\right|^{2} A_{i}\right)^{\frac{p}{m}} x, x\right\rangle^{\frac{m}{2}}-\left\langle\left(D_{i}\left|C_{i}^{*}\right|^{2} D_{i}^{*}\right)^{\frac{p}{m}} x, x\right\rangle^{\frac{m}{2}}\right)^{2}
\end{aligned} \\
& \leq \frac{n^{1-\frac{m}{r}}}{2^{\frac{m}{r}}} \sum_{i=1}^{n}\left(\frac{1}{2}\left\langle\left(A_{i}^{*}\left|B_{i}\right|^{2} A_{i}\right)^{\frac{r p}{m}} x, x\right\rangle+\left\langle\left(D_{i}\left|C_{i}^{*}\right|^{2} D_{i}^{*}\right)^{\frac{r p}{m}} x, x\right\rangle\right)^{\frac{m}{r}} \\
& -2^{-m} \sum_{i=1}^{n}\left(\left\langle\left(A_{i}^{*}\left|B_{i}\right|^{2} A_{i}\right)^{\frac{p}{m}} x, x\right\rangle^{\frac{m}{2}}-\left\langle\left(D_{i}\left|C_{i}^{*}\right|^{2} D_{i}^{*}\right)^{\frac{p}{m}} x, x\right\rangle^{\frac{m}{2}}\right)^{2}
\end{aligned}
$$

Theorem 4. Let $T_{i} \in \mathscr{B}(\mathscr{H})(i=1, \cdots, n), \alpha, \beta \geq 0$ such that $\alpha+\beta \geq 1, m \in \mathbb{N}$ and $r, p \geq m \geq 1$. Then

$$
w_{p}^{p}\left(T_{1}\left|T_{1}\right|^{\alpha+\beta-1}, \cdots, T_{n}\left|T_{n}\right|^{\alpha+\beta-1}\right) \leq \frac{n^{1-\frac{m}{r}}}{2^{\frac{m}{r}}}\left\|\sum_{i=1}^{n}\left(\left|T_{i}\right|^{\frac{2 \alpha r p}{m}}+\left|T_{i}^{*}\right|^{\frac{2 \beta r p}{m}}\right)\right\|^{\frac{m}{r}}-\inf _{\|x\|=1} \psi_{p, m, \alpha, \beta}(x),
$$

where

$$
\psi_{p, m, \alpha, \beta}(x):=2^{-m} \sum_{i=1}^{n}\left(\left\langle\left|T_{i}\right|^{\frac{2 \alpha p}{m}} x, x\right\rangle^{\frac{m}{2}}-\left\langle\left|T_{i}^{*}\right|^{\frac{2 \beta p}{m}} x, x\right\rangle^{\frac{m}{2}}\right)^{2} .
$$

for all $\alpha, \beta \geq 0$ such that $\alpha+\beta \geq 1$. 
Proof. Let $U_{i}$ be unitaries for all $i=1, \cdots, n$, setting $D_{i}=U_{i}, B=1_{\mathscr{H}}, C=\left|T_{i}\right|^{\beta}$ and $A_{i}=\left|T_{i}\right|^{\alpha}$ for all $\alpha, \beta \geq 0$ such that $\alpha+\beta \geq 1$ in (2.26), then we have

$$
D_{i} C_{i} B_{i} A_{i}=U_{i}\left|T_{i}\right|^{\beta}\left|T_{i}\right|^{\alpha}=U_{i}\left|T_{i}\right|\left|T_{i}\right|^{\alpha+\beta-1}=T_{i}\left|T_{i}\right|^{\alpha+\beta-1},
$$

also, we have $A_{i}^{*}\left|B_{i}\right|^{2} A_{i}=\left|T_{i}\right|^{2 \alpha}$ and $D_{i}\left|C_{i}^{*}\right|^{2} D_{i}^{*}=U_{i}\left|T_{i}\right|^{2 \beta} U_{i}^{*}=\left|T_{i}\right|^{2 \beta}$ for all $i=1, \cdots, n$.

Corollary 12. Let $T_{i} \in \mathscr{B}(\mathscr{H})(i=1, \cdots, n), \alpha, \beta \geq 0$ such that $\alpha+\beta \geq 1, m \in \mathbb{N}$ and $r, p \geq m \geq 1$. Then

$$
w_{p}^{p}\left(T_{1}\left|T_{1}\right|^{\alpha+\beta-1}, \cdots, T_{n}\left|T_{n}\right|^{\alpha+\beta-1}\right) \leq \frac{1}{2}\left\|\sum_{i=1}^{n}\left(\left|T_{i}\right|^{2 \alpha p}+\left|T_{i}^{*}\right|^{2 \beta p}\right)\right\|-\inf _{\|x\|=1} \psi_{p, 1, \alpha, \beta}(x),
$$

where

$$
\psi_{p, 1, \alpha, \beta}(x):=\frac{1}{2} \sum_{i=1}^{n}\left(\left\langle\left|T_{i}\right|^{2 \alpha p} x, x\right\rangle^{\frac{1}{2}}-\left\langle\left|T_{i}^{*}\right|^{2 \beta p} x, x\right\rangle^{\frac{1}{2}}\right)^{2} .
$$

for all $\alpha, \beta \geq 0$ such that $\alpha+\beta \geq 1$.

Proof. Setting $m=r=1$ in (2.27).

Corollary 13. Let $T_{i} \in \mathscr{B}(\mathscr{H})(i=1, \cdots, n)$. Then, for $m \in \mathbb{N}$ and $r, p \geq m \geq 1$,

$$
w_{p}^{p}\left(T_{1}, \cdots, T_{n}\right) \leq \frac{n^{1-\frac{m}{r}}}{2^{\frac{m}{r}}}\left\|\sum_{i=1}^{n}\left(\left|T_{i}\right|^{\frac{r p}{m}}+\left|T_{i}^{*}\right|^{\frac{r p}{m}}\right)\right\|^{\frac{m}{r}}-\inf _{\|x\|=1} \psi_{p, m, \frac{1}{2}, \frac{1}{2}}(x),
$$

where

$$
\psi_{p, m, \frac{1}{2}, \frac{1}{2}}(x):=2^{-m} \sum_{i=1}^{n}\left(\left\langle\left|T_{i}\right|^{\frac{p}{m}} x, x\right\rangle^{\frac{m}{2}}-\left\langle\left|T_{i}^{*}\right|^{\frac{p}{m}} x, x\right\rangle^{\frac{m}{2}}\right)^{2} .
$$

for all $\alpha, \beta \geq 0$ such that $\alpha+\beta \geq 1$.

Proof. Setting $\alpha=\beta=\frac{1}{2}$ in (2.27).

Corollary 14. Let $T_{i} \in \mathscr{B}(\mathscr{H})(i=1, \cdots, n)$. Then, for $m \in \mathbb{N}$ and $r, p \geq 1$,

$$
w_{p}^{p}\left(T_{1}, \cdots, T_{n}\right) \leq \frac{n^{1-\frac{1}{r}}}{2^{\frac{1}{r}}}\left\|\sum_{i=1}^{n}\left(\left|T_{i}\right|^{r p}+\left|T_{i}^{*}\right|^{r p}\right)\right\|^{\frac{1}{r}}-\inf _{\|x\|=1} \psi_{p, 1, \frac{1}{2}, \frac{1}{2}}(x),
$$

where

$$
\psi_{p, 1, \frac{1}{2}, \frac{1}{2}}(x):=\frac{1}{2} \sum_{i=1}^{n}\left(\left\langle\left|T_{i}\right|^{p} x, x\right\rangle^{\frac{1}{2}}-\left\langle\left|T_{i}^{*}\right|^{p} x, x\right\rangle^{\frac{1}{2}}\right)^{2} .
$$

for all $\alpha, \beta \geq 0$ such that $\alpha+\beta \geq 1$.

Proof. Setting $\alpha=\beta=\frac{1}{2}$ and $m=1$ in (2.29).

Corollary 15. Let $T_{i} \in \mathscr{B}(\mathscr{H})(i=1, \cdots, n)$. Then, for $m \in \mathbb{N}$ and $p \geq m \geq 1$,

$$
w_{p}^{p}\left(T_{1}, \cdots, T_{n}\right) \leq \frac{1}{2}\left\|\sum_{i=1}^{n}\left(\left|T_{i}\right|^{p}+\left|T_{i}^{*}\right|^{p}\right)\right\|-\inf _{\|x\|=1} \psi_{p, m, \frac{1}{2}, \frac{1}{2}}(x),
$$

where

$$
\psi_{p, m, \frac{1}{2}, \frac{1}{2}}(x):=2^{-m} \sum_{i=1}^{n}\left(\left\langle\left|T_{i}\right|^{\frac{p}{m}} x, x\right\rangle^{\frac{m}{2}}-\left\langle\left|T_{i}^{*}\right|^{\frac{p}{m}} x, x\right\rangle^{\frac{m}{2}}\right)^{2} .
$$

Proof. Setting $m=r$ in (2.29). 
Corollary 16. Let $T_{i} \in \mathscr{B}(\mathscr{H})(i=1, \cdots, n)$. Then, for $m \in \mathbb{N}$ and $r, p \geq m \geq 1$, we have

$$
w_{p}^{p}\left(T_{1}\left|T_{1}\right|, \cdots, T_{n}\left|T_{n}\right|\right) \leq \frac{n^{1-\frac{m}{r}}}{2^{\frac{m}{r}}}\left\|\sum_{i=1}^{n}\left(\left|T_{i}\right|^{\frac{2 r p}{m}}+\left|T_{i}^{*}\right|^{\frac{2 r p}{m}}\right)\right\|^{\frac{m}{r}}-\inf _{\|x\|=1} \psi_{p, m, 1,1}(x),
$$

where

$$
\psi_{p, m, 1,1}(x):=2^{-m} \sum_{i=1}^{n}\left(\left\langle\left|T_{i}\right|^{\frac{2 p}{m}} x, x\right\rangle^{\frac{m}{2}}-\left\langle\left|T_{i}^{*}\right|^{\frac{2 p}{m}} x, x\right\rangle^{\frac{m}{2}}\right)^{2} .
$$

for all $\alpha, \beta \geq 0$ such that $\alpha+\beta \geq 1$.

Proof. Setting $\alpha=\beta=1$ in (2.27).

Corollary 17. Let $B_{i}, C_{i}, U_{i}, V_{i} \in \mathscr{B}(\mathscr{H})(i=1, \cdots, n)$ such that $U_{i}, V_{i}$ unitaries. Then, for $m \in \mathbb{N}$ and $r, p \geq m \geq 1$, we have

$$
\begin{aligned}
& w_{p}^{p}\left(U_{1}^{*} C_{1} B_{1} U_{1}, \cdots, U_{n}^{*} C_{n} B_{n} U_{n}\right) \\
& \quad \leq \frac{n^{1-\frac{m}{r}}}{2^{\frac{m}{r}}}\left\|\sum_{i=1}^{n}\left(\left(U_{i}^{*}\left|B_{i}\right|^{2} U_{i}\right)^{\frac{r p}{m}}+\left(U_{i}^{*}\left|C_{i}^{*}\right|^{2} U_{i}\right)^{\frac{r p}{m}}\right)\right\|^{\frac{m}{r}}-\inf _{\|x\|=1} \xi_{p, m}(x),
\end{aligned}
$$

where

$$
\xi_{p, m}(x):=2^{-m} \sum_{i=1}^{n}\left(\left\langle\left(U_{i}^{*}\left|B_{i}\right|^{2} U_{i}\right)^{\frac{p}{m}} x, x\right\rangle^{\frac{m}{2}}-\left\langle\left(U_{i}^{*}\left|C_{i}^{*}\right|^{2} U_{i}\right)^{\frac{p}{m}} x, x\right\rangle^{\frac{m}{2}}\right)^{2} .
$$

Proof. Setting $A_{i}=U_{i}$ and $D_{i}=U_{i}^{*}$ in (2.26) and using the fact that .

Corollary 18. Let $A_{i}, D_{i} \in \mathscr{B}(\mathscr{H})(i=1, \cdots, n)$. Then, for $m \in \mathbb{N}$ and $r, p \geq m \geq 1$,

$$
w_{p}^{p}\left(D_{1} A_{1}, \cdots, D_{n} A_{n}\right) \leq \frac{n^{1-\frac{m}{r}}}{2^{\frac{m}{r}}}\left\|\sum_{i=1}^{n}\left(\left(A_{i}^{*} A_{i}\right)^{\frac{r p}{m}}+\left(D_{i} D_{i}^{*}\right)^{\frac{r p}{m}}\right)\right\|^{\frac{m}{r}}-\inf _{\|x\|=1} \xi_{p, m}(x),
$$

where

$$
\xi_{p, m}(x):=2^{-m} \sum_{i=1}^{n}\left(\left\langle\left(A_{i}^{*} A_{i}\right)^{\frac{p}{m}} x, x\right\rangle^{\frac{m}{2}}-\left\langle\left(D_{i} D_{i}^{*}\right)^{\frac{p}{m}} x, x\right\rangle^{\frac{m}{2}}\right)^{2} .
$$

Proof. Setting $B_{i}=C_{i}=1_{\mathscr{H}}$ for all $i=1, \cdots, n$ in (2.26).

Corollary 19. Let $A_{i}, D_{i} \in \mathscr{B}(\mathscr{H})(i=1, \cdots, n)$. Then, for $m \in \mathbb{N}$ and $r, p \geq m \geq 1$,

$$
w_{p}^{p}\left(A_{1}^{*} A_{1}, \cdots, A_{n}^{*} A_{n}\right) \leq n^{1-\frac{m}{r}}\left\|\sum_{i=1}^{n}\left(A_{i}^{*} A_{i}\right)^{\frac{r p}{m}}\right\|^{\frac{m}{r}} .
$$

In particular, for $m=r$

$$
w_{p}^{p}\left(A_{1}^{*} A_{1}, \cdots, A_{n}^{*} A_{n}\right) \leq\left\|\sum_{i=1}^{n}\left(A_{i}^{*} A_{i}\right)^{p}\right\| .
$$

Proof. Setting $D_{i}=A_{i}^{*}$ for all $i=1, \cdots, n$ in (2.34).

\section{UPPER AND LOWER BOUNDS FOR THE GENERALIZED EUCLIDEAN OPERATOR RADIUS}

In this section we provide some lower and upper bounds for the product of the generalized Euclidean operator radius. In order to to prove our results we need to recall the the following Hölder type inequality, which reads:

$$
\left(\sum_{j=1}^{n}\left|x_{j} y_{j}\right|^{r}\right)^{\frac{1}{r}} \leq\left(\sum_{j=1}^{n}\left|x_{j}\right|^{p}\right)^{\frac{1}{p}}\left(\sum_{j=1}^{n}\left|y_{j}\right|^{q}\right)^{\frac{1}{q}}
$$

for all complex numbers $x_{j}, y_{j}(1 \leq j \leq n)$ and all $p, q, r \geq 1$ such that $\frac{1}{p}+\frac{1}{q}=\frac{1}{r}$. 
Theorem 5. Let $D_{i}, C_{i}, B_{i}, A_{i} \in \mathscr{B}(\mathscr{H})(i=1, \cdots, n), r \geq 1$ and $p, q \geq 1$ with $\frac{1}{p}+\frac{1}{q}=\frac{1}{r}$. Then

$$
\begin{aligned}
& \frac{1}{n^{2 r-1}}\left\|\sum_{i=1}^{n} D_{i} C_{i} B_{i} A_{i}\right\|^{2 r} \\
& \leq w_{p}^{r}\left(A_{1}^{*}\left|B_{1}\right|^{2} A_{1}, \cdots, A_{n}^{*}\left|B_{n}\right|^{2} A_{n}\right) w_{q}^{r}\left(D_{1}\left|C_{1}^{*}\right|^{2} D_{1}^{*}, \cdots, D_{n}\left|C_{n}^{*}\right|^{2} D_{n}^{*}\right) \\
& \leq \max \left\{\frac{r}{p}, \frac{r}{q}\right\}\left\|\sum_{i=1}^{n}\left(A_{i}^{*}\left|B_{i}\right|^{2} A_{i}\right)^{p}+\left(D_{i}\left|C_{i}^{*}\right|^{2} D_{i}^{*}\right)^{q}\right\|-\inf _{\|x\|=1} \lambda(x, y),
\end{aligned}
$$

where,

$$
\lambda(x, y):=\min \left\{\frac{r}{p}, \frac{r}{q}\right\}\left(\sqrt{\sum_{i=1}^{n}\left\langle A_{i}^{*}\left|B_{i}\right|^{2} A_{i} x, y\right\rangle^{p}}-\sqrt{\sum_{i=1}^{n}\left\langle D_{i}\left|C_{i}^{*}\right|^{2} D_{i}^{*} x, y\right\rangle^{q}}\right)^{2}
$$

Proof. Let $x, y \in \mathscr{H}$. Applying inequality (3.1) and the convexity of $t^{2 r}$, we have

$$
\begin{aligned}
& \frac{1}{n^{2 r-1}}\left|\left\langle\left(\sum_{i=1}^{n} D_{i} C_{i} B_{i} A_{i}\right) x, y\right\rangle\right|^{2 r} \\
& =\frac{1}{n^{2 r-1}}\left|\sum_{i=1}^{n}\left\langle\left(D_{i} C_{i} B_{i} A_{i}\right) x, y\right\rangle\right|^{2 r} \\
& \leq \frac{1}{n^{2 r-1}}\left(\sum_{i=1}^{n}\left|\left\langle\left(D_{i} C_{i} B_{i} A_{i}\right) x, y\right\rangle\right|\right)^{2 r} \\
& \leq\left(\sum_{i=1}^{n}\left|\left\langle\left(D_{i} C_{i} B_{i} A_{i}\right) x, y\right\rangle\right|^{2 r}\right) \quad \text { (by Jensen's inequality) } \\
& \leq\left(\sum_{i=1}^{n}\left(\left\langle A_{i}^{*}\left|B_{i}\right|^{2} A_{i} x, y\right\rangle\left\langle D_{i}\left|C_{i}^{*}\right|^{2} D_{i}^{*} x, y\right\rangle\right)^{r}\right) \quad \text { (by (2.6)) } \\
& \leq\left(\sum_{i=1}^{n}\left\langle A_{i}^{*}\left|B_{i}\right|^{2} A_{i} x, y\right\rangle^{p}\right)^{\frac{r}{p}}\left(\sum_{i=1}^{n}\left\langle D_{i}\left|C_{i}^{*}\right|^{2} D_{i}^{*} x, y\right\rangle^{q}\right)^{\frac{r}{q}} \\
& \leq \frac{r}{p} \sum_{i=1}^{n}\left\langle A_{i}^{*}\left|B_{i}\right|^{2} A_{i} x, y\right\rangle^{p}+\frac{r}{q} \sum_{i=1}^{n}\left\langle D_{i}\left|C_{i}^{*}\right|^{2} D_{i}^{*} x, y\right\rangle^{q} \quad \text { by (2.4) } \\
& -\min \left\{\frac{r}{p}, \frac{r}{q}\right\}\left(\sqrt{\sum_{i=1}^{n}\left\langle A_{i}^{*}\left|B_{i}\right|^{2} A_{i} x, y\right\rangle^{p}}-\sqrt{\sum_{i=1}^{n}\left\langle D_{i}\left|C_{i}^{*}\right|^{2} D_{i}^{*} x, y\right\rangle^{q}}\right)^{2} \\
& \leq \frac{r}{p} \sum_{i=1}^{n}\left\langle\left(A_{i}^{*}\left|B_{i}\right|^{2} A_{i}\right)^{p} x, y\right\rangle+\frac{r}{q} \sum_{i=1}^{n}\left\langle\left(D_{i}\left|C_{i}^{*}\right|^{2} D_{i}^{*}\right)^{q} x, y\right\rangle \quad \text { (by McCarthy inequality) } \\
& -\min \left\{\frac{r}{p}, \frac{r}{q}\right\}\left(\sqrt{\sum_{i=1}^{n}\left\langle A_{i}^{*}\left|B_{i}\right|^{2} A_{i} x, y\right\rangle^{p}}-\sqrt{\sum_{i=1}^{n}\left\langle D_{i}\left|C_{i}^{*}\right|^{2} D_{i}^{*} x, y\right\rangle^{q}}\right)^{2}
\end{aligned}
$$

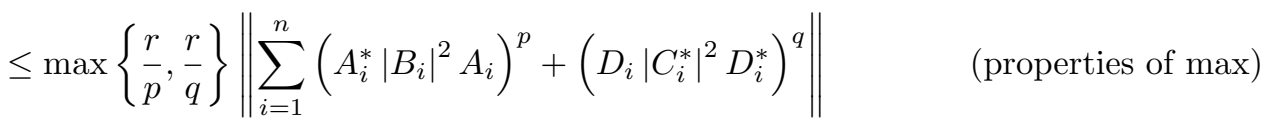

$$
\begin{aligned}
& -\min \left\{\frac{r}{p}, \frac{r}{q}\right\}\left(\sqrt{\sum_{i=1}^{n}\left\langle A_{i}^{*}\left|B_{i}\right|^{2} A_{i} x, y\right\rangle^{p}}-\sqrt{\sum_{i=1}^{n}\left\langle D_{i}\left|C_{i}^{*}\right|^{2} D_{i}^{*} x, y\right\rangle^{q}}\right)^{2}
\end{aligned}
$$


Taking the supremum over $x, y \in \mathscr{H}$ with $\|x\|=\|y\|=1$, then the left and right hand side follows immediately the middle term of the inequality follows by (3.3), and thus the desired result is obtained.

Corollary 20. Let $D_{i}, C_{i}, B_{i}, A_{i} \in \mathscr{B}(\mathscr{H})(i=1, \cdots, n), r \geq 1$. Then

$$
\begin{aligned}
& \frac{1}{n}\left\|\sum_{i=1}^{n} D_{i} C_{i} B_{i} A_{i}\right\|^{2} \\
& \leq w_{\mathrm{e}}\left(A_{1}^{*}\left|B_{1}\right|^{2} A_{1}, \cdots, A_{n}^{*}\left|B_{n}\right|^{2} A_{n}\right) w_{\mathrm{e}}\left(D_{1}\left|C_{1}^{*}\right|^{2} D_{1}^{*}, \cdots, D_{n}\left|C_{n}^{*}\right|^{2} D_{n}^{*}\right) \\
& \leq \frac{1}{2}\left\|\sum_{i=1}^{n}\left\{\left(A_{i}^{*}\left|B_{i}\right|^{2} A_{i}\right)^{2}+\left(D_{i}\left|C_{i}^{*}\right|^{2} D_{i}^{*}\right)^{2}\right\}\right\|-\inf _{\|x\|=\|y\|=1} \lambda(x, y),
\end{aligned}
$$

where

$$
\lambda(x, y):=\frac{1}{2}\left(\sqrt{\sum_{i=1}^{n}\left\langle A_{i}^{*}\left|B_{i}\right|^{2} A_{i} x, y\right\rangle^{2}}-\sqrt{\sum_{i=1}^{n}\left\langle D_{i}\left|C_{i}^{*}\right|^{2} D_{i}^{*} x, y\right\rangle^{2}}\right)^{2}
$$

Proof. Setting $p=q=2$ and $r=1$ in (3.2) we get the desired result.

Corollary 21. Let $D_{i}, A_{i} \in \mathscr{B}(\mathscr{H})(i=1, \cdots, n), r \geq 1$ and $p, q \geq 1$ with $\frac{1}{p}+\frac{1}{q}=\frac{1}{r}$. Then

$$
\begin{aligned}
\frac{1}{n^{2 r-1}}\left\|\sum_{i=1}^{n} D_{i} A_{i}\right\|^{2 r} & \leq w_{p}^{r}\left(A_{1}^{*} A_{1}, \cdots, A_{n}^{*} A_{n}\right) w_{q}^{r}\left(D_{1} D_{1}^{*}, \cdots, D_{n} D_{n}^{*}\right) \\
& \leq \max \left\{\frac{r}{p}, \frac{r}{q}\right\}\left\|\sum_{i=1}^{n}\left(A_{i}^{*} A_{i}\right)^{p}+\left(D_{i} D_{i}^{*}\right)^{q}\right\|-\inf _{\|x\|=1} \lambda(x, y),
\end{aligned}
$$

where,

$$
\lambda(x, y):=\min \left\{\frac{r}{p}, \frac{r}{q}\right\}\left(\sqrt{\sum_{i=1}^{n}\left\langle A_{i}^{*}\left|B_{i}\right|^{2} A_{i} x, y\right\rangle^{p}}-\sqrt{\sum_{i=1}^{n}\left\langle D_{i}\left|C_{i}^{*}\right|^{2} D_{i}^{*} x, y\right\rangle^{q}}\right)^{2}
$$

Proof. Setting $B_{i}=C_{i}=1_{\mathscr{H}}$ in (3.2) we get the required result.

Corollary 22. Let $D_{i}, A_{i} \in \mathscr{B}(\mathscr{H})(i=1, \cdots, n), r \geq 1$ and $p, q \geq 1$ with $\frac{1}{p}+\frac{1}{q}=\frac{1}{r}$. Then

$$
\begin{aligned}
\frac{1}{n^{2 r-1}}\left\|\sum_{i=1}^{n} A_{i}^{*} A_{i}\right\|^{2 r} & \leq w_{p}^{r}\left(A_{1}^{*} A_{1}, \cdots, A_{n}^{*} A_{n}\right) w_{q}^{r}\left(A_{i} A_{i}^{*}, \cdots, A_{i} A_{i}^{*}\right) \\
& \leq \max \left\{\frac{r}{p}, \frac{r}{q}\right\}\left\|\sum_{i=1}^{n}\left(A_{i}^{*} A_{i}\right)^{p}+\left(A_{i} A_{i}^{*}\right)^{q}\right\|-\inf _{\|x\|=1} \lambda(x, y),
\end{aligned}
$$

where,

$$
\lambda(x, y):=\min \left\{\frac{r}{p}, \frac{r}{q}\right\}\left(\sqrt{\sum_{i=1}^{n}\left\langle A_{i}^{*} A_{i} x, y\right\rangle^{p}}-\sqrt{\sum_{i=1}^{n}\left\langle A_{i} A_{i}^{*} x, y\right\rangle^{q}}\right)^{2}
$$

Proof. Setting $D_{i}=A_{i}$ and $B_{i}=C_{i}=1_{\mathscr{H}}$ in (3.5).

Corollary 23. Let $D_{i}, A_{i} \in \mathscr{B}(\mathscr{H})(i=1, \cdots, n), r \geq 1$ and $p, q \geq 1$ with $\frac{1}{p}+\frac{1}{q}=\frac{1}{r}$. Then

$$
\frac{1}{n}\left\|\sum_{i=1}^{n} A_{i}^{2}\right\|^{2} \leq w_{\mathrm{e}}^{2}\left(A_{1}^{*} A_{1}, \cdots, A_{n}^{*} A_{n}\right) \leq \frac{1}{2}\left\|\sum_{i=1}^{n}\left(A_{i}^{*} A_{i}\right)^{2}+\left(A_{i} A_{i}^{*}\right)^{2}\right\|-\inf _{\|x\|=\|y\|=1} \lambda(x, y),
$$


where,

$$
\lambda(x, y):=\frac{1}{2}\left(\sqrt{\sum_{i=1}^{n}\left\langle A_{i}^{*} A_{i} x, y\right\rangle^{2}}-\sqrt{\sum_{i=1}^{n}\left\langle A_{i} A_{i}^{*} x, y\right\rangle^{2}}\right)^{2}
$$

Proof. Setting $p=q=2$ and $r=1$ in (3.6).

Theorem 6. Let $T_{i} \in \mathscr{B}(\mathscr{H})(i=1, \cdots, n), r \geq 1, p, q \geq 1$ with $\frac{1}{p}+\frac{1}{q}=\frac{1}{r}$ and $\alpha, \beta \geq 0$ such that $\alpha+\beta \geq 1$. Then

$$
\begin{aligned}
\frac{1}{n^{2 r-1}}\left\|\sum_{i=1}^{n} T_{i}\left|T_{i}\right|^{\alpha+\beta-1}\right\|^{2 r} & \leq w_{p}^{r}\left(\left|T_{1}\right|^{2 \alpha}, \cdots,\left|T_{n}\right|^{2 \alpha}\right) w_{q}^{r}\left(\left|T_{1}^{*}\right|^{2 \beta}, \cdots,\left|T_{n}^{*}\right|^{2 \beta}\right) \\
& \leq \max \left\{\frac{r}{p}, \frac{r}{q}\right\}\left\|\sum_{i=1}^{n}\left|T_{i}\right|^{2 p \alpha}+\left|T_{i}^{*}\right|^{2 q \beta}\right\|-\inf _{\|x\|=\|y\|=1} \lambda(x, y),
\end{aligned}
$$

where,

$$
\lambda(x, y):=\min \left\{\frac{r}{p}, \frac{r}{q}\right\}\left(\sqrt{\sum_{i=1}^{n}\left\langle\left|T_{i}\right|^{2 \alpha} x, y\right\rangle^{p}}-\sqrt{\sum_{i=1}^{n}\left\langle\left|T_{i}^{*}\right|^{2 \beta} x, y\right\rangle^{q}}\right)^{2}
$$

Proof. Let $U_{i}$ be unitaries for all $i=1, \cdots, n$, setting $D_{i}=U_{i}, B=1_{\mathscr{H}}, C=\left|T_{i}\right|^{\beta}$ and $A_{i}=\left|T_{i}\right|^{\alpha}$ for all $\alpha, \beta \geq 0$ such that $\alpha+\beta \geq 1$ in (3.2), then we have

$$
D_{i} C_{i} B_{i} A_{i}=U_{i}\left|T_{i}\right|^{\beta}\left|T_{i}\right|^{\alpha}=U_{i}\left|T_{i}\right|\left|T_{i}\right|^{\alpha+\beta-1}=T_{i}\left|T_{i}\right|^{\alpha+\beta-1},
$$

also, we have $A_{i}^{*}\left|B_{i}\right|^{2} A_{i}=\left|T_{i}\right|^{2 \alpha}$ and $D_{i}\left|C_{i}^{*}\right|^{2} D_{i}^{*}=U_{i}\left|T_{i}\right|^{2 \beta} U_{i}^{*}=\left|T_{i}\right|^{2 \beta}$ for all $i=1, \cdots, n$.

Corollary 24. Let $T_{i} \in \mathscr{B}(\mathscr{H})(i=1, \cdots, n), r \geq 1$ and $p, q \geq 1$ with $\frac{1}{p}+\frac{1}{q}=\frac{1}{r}$. Then

$$
\begin{aligned}
\frac{1}{n^{2 r-1}}\left\|\sum_{i=1}^{n} T_{i}\right\|^{2 r} & \leq w_{p}^{r}\left(\left|T_{1}\right|, \cdots,\left|T_{n}\right|\right) w_{q}^{r}\left(\left|T_{1}^{*}\right|, \cdots,\left|T_{n}^{*}\right|\right) \\
& \leq \max \left\{\frac{r}{p}, \frac{r}{q}\right\}\left\|\sum_{i=1}^{n}\left|T_{i}\right|^{p}+\left|T_{i}^{*}\right|^{q}\right\|-\inf _{\|x\|=\|y\|=1} \lambda(x, y),
\end{aligned}
$$

where,

$$
\lambda(x, y):=\min \left\{\frac{r}{p}, \frac{r}{q}\right\}\left(\sqrt{\sum_{i=1}^{n}\left\langle\left|T_{i}\right| x, y\right\rangle^{p}}-\sqrt{\sum_{i=1}^{n}\left\langle\left|T_{i}^{*}\right| x, y\right\rangle^{q}}\right)^{2}
$$

Proof. Setting $\alpha=\beta=\frac{1}{2}$ in (3.8).

Corollary 25. Let $T_{i} \in \mathscr{B}(\mathscr{H})(i=1, \cdots, n), \alpha, \beta \geq 0$ such that $\alpha+\beta \geq 1$. Then

$$
\begin{aligned}
\frac{1}{n}\left\|\sum_{i=1}^{n} T_{i}\left|T_{i}\right|^{\alpha+\beta-1}\right\|^{2} & \leq w_{\mathrm{e}}\left(\left|T_{1}\right|^{2 \alpha}, \cdots,\left|T_{n}\right|^{2 \alpha}\right) w_{\mathrm{e}}\left(\left|T_{1}^{*}\right|^{2 \beta}, \cdots,\left|T_{n}^{*}\right|^{2 \beta}\right) \\
& \leq \frac{1}{2}\left\|\sum_{i=1}^{n}\left|T_{i}\right|^{4 \alpha}+\left|T_{i}^{*}\right|^{4 \beta}\right\|-\inf _{\|x\|=\|y\|=1} \lambda(x, y),
\end{aligned}
$$

where,

$$
\lambda(x, y):=\frac{1}{2}\left(\sqrt{\sum_{i=1}^{n}\left\langle\left|T_{i}\right|^{2 \alpha} x, y\right\rangle^{2}}-\sqrt{\sum_{i=1}^{n}\left\langle\left|T_{i}^{*}\right|^{2 \beta} x, y\right\rangle^{2}}\right)^{2}
$$

Proof. Setting $p=q=2$ and $r=1$ in (3.8). 
Corollary 26. Let $T_{i} \in \mathscr{B}(\mathscr{H})(i=1, \cdots, n)$. Then

$$
\begin{aligned}
\frac{1}{n}\left\|\sum_{i=1}^{n} T_{i}\right\|^{2} & \leq w_{\mathrm{e}}\left(\left|T_{1}\right|, \cdots,\left|T_{n}\right|\right) w_{\mathrm{e}}\left(\left|T_{1}^{*}\right|, \cdots,\left|T_{n}^{*}\right|\right) \\
& \leq \frac{1}{2}\left\|\sum_{i=1}^{n} T_{i}^{*} T_{i}+T_{i} T_{i}^{*}\right\|-\inf _{\|x\|=\|y\|=1} \lambda(x, y),
\end{aligned}
$$

where,

$$
\lambda(x, y):=\frac{1}{2}\left(\sqrt{\sum_{i=1}^{n}\left\langle\left|T_{i}\right| x, y\right\rangle^{2}}-\sqrt{\sum_{i=1}^{n}\left\langle\left|T_{i}^{*}\right| x, y\right\rangle^{2}}\right)^{2}
$$

Proof. Setting $\alpha=\beta=\frac{1}{2}$ in (3.10).

We note that, in 2005, Kittaneh in [16] proved that

$$
\frac{1}{4}\left\|T^{*} T+T T^{*}\right\| \leq w^{2}(T) \leq \frac{1}{2}\left\|T^{*} T+T T^{*}\right\|
$$

for Hilbert space operator $T \in \mathscr{B}(\mathscr{H})$. These inequalities are sharp. These inequalities were also reformulated and generalized in [10] but in terms of the Cartesian decomposition.

In 2009, Popescu [21] proved that

$$
\frac{1}{2 \sqrt{n}}\left\|\sum_{k=1}^{n} T_{k} T_{k}^{*}\right\|^{\frac{1}{2}} \leq w_{\mathrm{e}}\left(T_{1}, \cdots, T_{n}\right) \leq\left\|\sum_{k=1}^{n} T_{k} T_{k}^{*}\right\|^{\frac{1}{2}}
$$

As noted in [20], and as a special case of (3.13); if $A=B+i C$ is the Cartesian decomposition of $A$, then

$$
w_{\mathrm{e}}^{2}(B, C)=\sup _{\|x\|=1}\left\{|\langle B x, x\rangle|^{2}+|\langle C x, x\rangle|^{2}\right\}=\sup _{\|x\|=1}|\langle A x, x\rangle|^{2}=w^{2}(A) .
$$

But since $A^{*} A+A A^{*}=2\left(B^{2}+C^{2}\right)$, then we have

$$
\frac{1}{16}\left\|T^{*} T+T T^{*}\right\| \leq w^{2}(T) \leq \frac{1}{2}\left\|T^{*} T+T T^{*}\right\| .
$$

It should be noted here that, the case when $n=2$, was also studied by Dragomir in [9] where he obtained some very interesting results regading Euclidean operator radius of two operators $w_{\mathrm{e}}\left(T_{1}, T_{2}\right)$.

Next, we give a generalization of (3.12) and refine (indeed improve) (3.13) (and thus (3.14)) to the generalized Euclidean operator radius.

Theorem 7. Let $T_{k} \in \mathscr{B}(\mathscr{H})(k=1, \cdots, n)$. Then

$$
\frac{1}{2^{p+1} n^{p-1}}\left\|\sum_{k=1}^{n} T_{k}^{*} T_{k}+T_{k} T_{k}^{*}\right\|^{p} \leq w_{2 p}^{p}\left(T_{1}, \cdots, T_{n}\right) \leq \frac{1}{2^{p}}\left\|\sum_{k=1}^{n}\left(T_{k}^{*} T_{k}+T_{k} T_{k}^{*}\right)^{p}\right\|
$$

for all $p \geq 1$.

Proof. Let $B_{k}+i C_{k}$ be the Cartesian decomposition of $T_{k}$ for all $k=1, \cdots, n$. As in the proof of (3.12) in [16], we have

$$
\begin{aligned}
\left|\left\langle T_{k} x, x\right\rangle\right|^{2 p} & =\left(\left\langle B_{k} x, x\right\rangle^{2}+\left\langle C_{k} x, x\right\rangle^{2}\right)^{p} \\
& \geq \frac{1}{2^{p}}\left(\left|\left\langle B_{k} x, x\right\rangle\right|+\left|\left\langle C_{k} x, x\right\rangle\right|\right)^{2 p} \\
& \geq \frac{1}{2^{p}}\left|\left\langle B_{k} x, x\right\rangle+\left\langle C_{k} x, x\right\rangle\right|^{2 p} \\
& =\frac{1}{2^{p}}\left|\left\langle B_{k} \pm C_{k} x, x\right\rangle\right|^{2 p} .
\end{aligned}
$$


Summing over $k$ and then taking the supremum over all unit vector $x \in \mathscr{H}$, we get

$$
\begin{aligned}
w_{2 p}^{p}\left(T_{1}, \cdots, T_{n}\right) & \geq \frac{1}{2^{p}} \sup _{\|x\|=1} \sum_{k=1}^{n}\left|\left\langle B_{k} \pm C_{k} x, x\right\rangle\right|^{2 p} \\
& \geq \frac{1}{2^{p}} \frac{1}{n^{p-1}} \sup _{\|x\|=1}\left(\sum_{k=1}^{n}\left|\left\langle B_{k} \pm C_{k} x, x\right\rangle\right|^{2}\right)^{p} \quad \text { (by Jensen's inequality) } \\
& =\frac{1}{2^{p}} \frac{1}{n^{p-1}}\left\|\sum_{k=1}^{n}\left(B_{k} \pm C_{k}\right)^{2}\right\|^{p} .
\end{aligned}
$$

Thus,

$$
\begin{aligned}
2 w_{2 p}^{p}\left(T_{1}, \cdots, T_{n}\right) & \geq \frac{1}{2^{p}} \frac{1}{n^{p-1}}\left\|\sum_{k=1}^{n}\left(B_{k}+C_{k}\right)^{2}\right\|^{p}+\frac{1}{2^{p}} \frac{1}{n^{p-1}}\left\|\sum_{k=1}^{n}\left(B_{k}-C_{k}\right)^{2}\right\|^{p} \\
& \geq \frac{1}{2^{p}} \frac{1}{n^{p-1}}\left\|\sum_{k=1}^{n}\left(B_{k}+C_{k}\right)^{2}+\sum_{k=1}^{n}\left(B_{k}-C_{k}\right)^{2}\right\|^{p} \\
& =\frac{1}{2^{p}} \frac{1}{n^{p-1}}\left\|\sum_{k=1}^{n}\left\{\left(B_{k}+C_{k}\right)^{2}+\left(B_{k}-C_{k}\right)^{2}\right\}\right\|^{p} \\
& =\frac{1}{n^{p-1}}\left\|\sum_{k=1}^{n} B_{k}^{2}+C_{k}^{2}\right\|^{p} \\
& =\frac{1}{n^{p-1}}\left\|\sum_{k=1}^{n} \frac{T_{k}^{*} T_{k}+T_{k} T_{k}^{*}}{2}\right\|^{p} \\
& =\frac{1}{2^{p} n^{p-1}}\left\|\sum_{k=1}^{n} T_{k}^{*} T_{k}+T_{k} T_{k}^{*}\right\|^{p}
\end{aligned}
$$

and hence,

$$
w_{2 p}^{p}\left(T_{1}, \cdots, T_{n}\right) \geq \frac{1}{2^{p+1} n^{p-1}}\left\|\sum_{k=1}^{n} T_{k}^{*} T_{k}+T_{k} T_{k}^{*}\right\|^{p},
$$

which proves the left hand side of the inequality in (3.15).

To prove the second inequality, for every unit vector $x \in \mathscr{H}$ we have

$$
\begin{aligned}
\sum_{k=1}^{n}\left|\left\langle T_{k} x, x\right\rangle\right|^{2 p} & =\sum_{k=1}^{n}\left(\left\langle B_{k} x, x\right\rangle^{2}+\left\langle C_{k} x, x\right\rangle^{2}\right)^{p} \\
& \leq \sum_{k=1}^{n}\left(\left\langle B_{k}^{2} x, x\right\rangle+\left\langle C_{k}^{2} x, x\right\rangle\right)^{p} \\
& =\sum_{k=1}^{n}\left\langle\left(B_{k}^{2}+C_{k}^{2}\right) x, x\right\rangle^{p}
\end{aligned}
$$

which implies that

$$
\begin{aligned}
\sup _{\|x\|=1} \sum_{k=1}^{n}\left|\left\langle T_{k} x, x\right\rangle\right|^{2 p}=w_{2 p}^{p}\left(T_{1}, \cdots, T_{1}\right) & \leq \sup _{\|x\|=1} \sum_{k=1}^{n}\left\langle\left(B_{k}^{2}+C_{k}^{2}\right) x, x\right\rangle^{p} \\
& =\left\|\sum_{k=1}^{n}\left(B_{k}^{2}+C_{k}^{2}\right)^{p}\right\| \\
& =\frac{1}{2^{p}}\left\|\sum_{k=1}^{n}\left(T_{k}^{*} T_{k}+T_{k} T_{k}^{*}\right)^{p}\right\|,
\end{aligned}
$$


which proves the right hand side of (3.15).

Remark 2. Clearly, by setting $n=1$ in (3.15) we recapture (3.12).

A very interesting case of (3.15) is considered in the following corollary.

Corollary 27. Let $T, S \in \mathscr{B}(\mathscr{H})$. Then

$$
\begin{aligned}
\frac{1}{2^{2 p}}\left\|T^{*} T+T T^{*}+S^{*} S+S S^{*}\right\|^{p} & \leq w_{2 p}^{p}(T, S) \\
& \leq \frac{1}{2^{p}}\left\|\left(T^{*} T+T T^{*}\right)^{p}+\left(S^{*} S+S S^{*}\right)^{p}\right\|
\end{aligned}
$$

for all $p \geq 1$.

Proof. Setting $n=2$ in (3.15).

Remark 3. In particular, setting $p=1$ in (3.16) we get

$$
\begin{aligned}
\frac{1}{4}\left\|T^{*} T+T T^{*}+S^{*} S+S S^{*}\right\| & \leq w_{\mathrm{e}}(T, S) \\
& \leq \frac{1}{2}\left\|T^{*} T+T T^{*}+S^{*} S+S S^{*}\right\| .
\end{aligned}
$$

Moreover, if we choose $T=S$, then

$$
\frac{1}{2}\left\|T^{*} T+T T^{*}\right\| \leq w_{\mathrm{e}}(T, T) \leq\left\|T^{*} T+T T^{*}\right\| .
$$

Remark 4. A lower and upper bounds for the Rhombic numerical radius could be deduced as follows:

In (1.4) the inequality holds for any $p \geq 1$. Setting $p=2 q$, then (1.4) reduces to

$$
w_{2 q}\left(T_{1}, \cdots, T_{n}\right) \leq w_{R}\left(T_{1}, \cdots, T_{n}\right) \leq n^{1-\frac{1}{2 q}} w_{2 q}\left(T_{1}, \cdots, T_{n}\right) .
$$

which implies that

$$
w_{2 q}^{q}\left(T_{1}, \cdots, T_{n}\right) \leq w_{R}^{q}\left(T_{1}, \cdots, T_{n}\right) \leq n^{q-\frac{1}{2}} w_{2 q}^{q}\left(T_{1}, \cdots, T_{n}\right) .
$$

Combining the inequalities (3.17) with (3.15) we get

$$
\begin{aligned}
\frac{1}{2^{2 q+1} n^{q-1}}\left\|\sum_{k=1}^{n} T_{k}^{*} T_{k}+T_{k} T_{k}^{*}\right\|^{q} & \leq w_{2 q}^{q}\left(T_{1}, \cdots, T_{n}\right) \\
& \leq w_{R}^{q}\left(T_{1}, \cdots, T_{n}\right) \\
& \leq n^{q-\frac{1}{2}} w_{2 q}^{q}\left(T_{1}, \cdots, T_{n}\right) \\
& \leq \frac{n^{q-\frac{1}{2}}}{2^{q}}\left\|\sum_{k=1}^{n}\left(T_{k}^{*} T_{k}+T_{k} T_{k}^{*}\right)^{q}\right\|
\end{aligned}
$$

for any $q \geq \frac{1}{2}$.

\section{REFERENCES}

[1] S. Abramovich, G. Jameson and G. Sinnamon, Refining Jensen's inequality, Bull. Math. Soc. Sci. Math. Roumanie, 47 (2004), 3-14.

[2] M.W. Alomari, On the generalized mixed Schwarz inequality, Proceedings of the Institute of Mathematics and Mechanics, National Academy of Sciences of Azerbaijan, to appear

[3] M.W. Alomari, Refinements of some numerical radius inequalities for Hilbert space operators, Linear and Multilinear Algebra, (2019), DOI: 10.1080/03081087.2019.1624682

[4] M.W. Alomari, Improvements of some numerical radius inequalities, ArXiv, (2019). DOI:10.1080/03081087.2019.1624682

[5] A.B. Bajmaeh and M.E. Omidvar, Some Inequalities for the numerical radius and Rhombic numerical radius, Kragujevac Journal of Mathematics 42 (4) (2018), 569-577.

[6] S.S. Dragomir, Inequalities for the numerical radius of linear operators in Hilbert spaces, SpringerBriefs in Mathematics, 2013.

[7] S.S. Dragomir, Some inequalities for the norm and the numerical radius of linear operator in Hilbert spaces, Tamkang J. Math., 39 (1) (2008), 1-7.

[8] S.S. Dragomir, Some inequalities generalizing Kato's and Furuta's results, FILOMAT, 28 (1) (2014), $179-195$. 
[9] S.S. Dragomir, Some inequalities for the Euclidean operator radius of two operators in Hilbert spaces, Linear Algebra Appl., 419 (2006), 256-264.

[10] M. El-Haddad and F. Kittaneh, Numerical radius inequalities for Hilbert space operators. II. Studia Math., 182 (2) (2007), $133-140$

[11] T. Furuta, An extension of the Heinz-Kato theorem, Proc. Amer. Math. Soc., 120 (1994), no. 3, 785-787.

[12] M. Hajmohamadi, R. Lashkaripour and M. Bakherad, Some generalizations of numerical radius on off-diagonal part of $2 \times 2$ operator matrices, Journal of Mathematical Inequalities, 12 (2) (2018), 447-457.

[13] M. Hajmohamadi, R. Lashkaripour and M. Bakherad, Further refinements of generalized numerical radius inequalities for Hilbert space operators, Georgian Mathematical Journal, 2019, DOI: https://doi.org/10.1515/gmj-2019-2023

[14] P.R. Halmos, A Hilbert space problem book, Van Nostrand Company, Inc., Princeton, N.J., 1967.

[15] T. Kato, Notes on some inequalities for linear operators, Math. Ann., 125 (1952), 208-212.

[16] F. Kittaneh, Numerical radius inequalities for Hilbert space operators, Studia Math., 168 (1) (2005), 73-80.

[17] F. Kittaneh and Y. Manasrah, Improved Young and Heinz inequalities for matrices, J. Math. Anal. Appl. 361 (2010), $262-269$.

[18] F. Kittaneh, Notes on some inequalities for Hilbert Space operators, Publ. Res. Inst. Math. Sci., 24 (2) (1988), $283-293$.

[19] C.-S. Lin and S.S. Dragomir, On High-power Operator inequalities and spectral radii of operators, Publ. RIMS, Kyoto Univ., 42 (2006), 391-397.

[20] M.S. Moslehian, M. Sattari, K. Shebrawi, Extension of Euclidean operator radius inequalities, Mathematica Scandinavica, 120 (1) (2017), 129-144.

[21] G. Popescu, Unitary invariants in multivariable operator theory, Mem. Amer. Math. Soc., Vol. 200, no $941,2009$.

[22] W. Reid, Symmetrizable completely continuous linear tarnsformations in Hilbert space, Duke Math., 18 (1951), $41-56$.

[23] M. Sattari, M.S. Moslehian and T. Yamazaki, Some genaralized numerical radius inequalities for Hilbert space operators, Linear Algebra Appl., 470 (2014), 1-12.

[24] A. Sheikhhosseini, M. S. Moslehian and K. Shebrawi, Inequalities for generalized Euclidean operator radius via Young's inequality, J. Math. Anal. Appl. 445 (2017), 1516-1529.

Department of Mathematics, Faculty of Science and Information Technology, Irbid National University, P.O.

Box 2600, Irbid, P.C. 21110, Jordan.

Email address: mwomath@gmail.com 